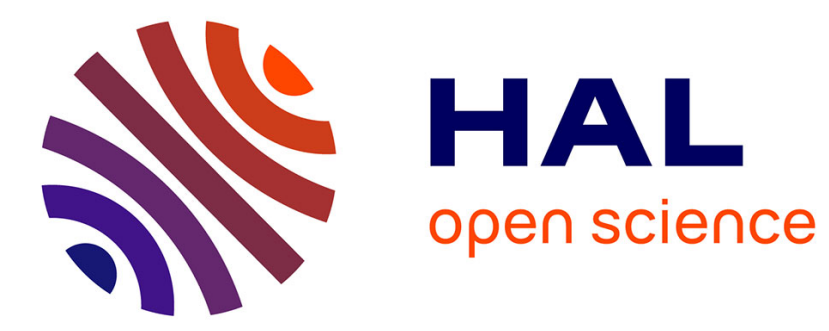

\title{
Unlocking the beamforming potential of LoRa for long-range multi-target respiration sensing
}

Fusang Zhang, Zhaoxin Chang, Jie Xiong, Rong Zheng, Junqi Ma, Kai Niu, Beihong Jin, Daqing Zhang

\section{- To cite this version:}

Fusang Zhang, Zhaoxin Chang, Jie Xiong, Rong Zheng, Junqi Ma, et al.. Unlocking the beamforming potential of LoRa for long-range multi-target respiration sensing. Proceedings of the ACM on Interactive, Mobile, Wearable and Ubiquitous Technologies , 2021, 5 (2), pp.85:1-85:25. 10.1145/3463526 . hal-03363386

\section{HAL Id: hal-03363386 https://hal.science/hal-03363386}

Submitted on 3 Oct 2021

HAL is a multi-disciplinary open access archive for the deposit and dissemination of scientific research documents, whether they are published or not. The documents may come from teaching and research institutions in France or abroad, or from public or private research centers.
L'archive ouverte pluridisciplinaire HAL, est destinée au dépôt et à la diffusion de documents scientifiques de niveau recherche, publiés ou non, émanant des établissements d'enseignement et de recherche français ou étrangers, des laboratoires publics ou privés. 


\section{Unlocking the Beamforming Potential of LoRa for Long-range Multi-target Respiration Sensing}

FUSANG ZHANG, Institute of Software, Chinese Academy of Sciences and University of Chinese Academy of Sciences, China ZHAOXIN CHANG, Institut Polytechnique de Paris, France

JIE XIONG, University of Massachusetts Amherst, USA

RONG ZHENG, McMaster University, Canada

JUNQI MA, Beijing University of Posts and Telecommunications, China

KAI NIU, Peking University, China

BEIHONG JIN, Institute of Software, Chinese Academy of Sciences and University of Chinese Academy of Sciences, China

DAQING ZHANG, Institut Polytechnique de Paris, France and Peking University, China

Despite extensive research effort in contact-free sensing using RF signals in the last few years, there still exist significant barriers preventing their wide adoptions. One key issue is the inability to sense multiple targets due to the intrinsic nature of relying on reflection signals for sensing: the reflections from multiple targets get mixed at the receiver and it is extremely difficult to separate these signals to sense each individual. This problem becomes even more severe in long-range LoRa sensing because the sensing range is much larger compared to WiFi and acoustic based sensing. In this work, we address the challenging multi-target sensing issue, moving LoRa sensing one big step towards practical adoption. The key idea is to effectively utilize multiple antennas at the LoRa gateway to enable spatial beamforming to support multi-target sensing. While traditional beamforming methods adopted in WiFi and Radar systems rely on accurate channel information or transmitterreceiver synchronization, these requirements can not be satisfied in LoRa systems: the transmitter and receiver are not synchronized and no channel state information can be obtained from the cheap LoRa nodes. Another interesting observation is that while beamforming helps to increase signal strength, the phase/amplitude information which is critical for sensing can get corrupted during the beamforming process, eventually compromising the sensing capability. In this paper, we propose novel signal processing methods to address the issues above to enable long-range multi-target reparation sensing with LoRa. Extensive experiments show that our system can monitor the respiration rates of five human targets simultaneously at an average accuracy of $98.1 \%$.

CCS Concepts: • Human-centered computing $\rightarrow$ Ubiquitous and mobile computing systems and tools.

Authors' addresses: F. Zhang, B. Jin, State Key Laboratory of Computer Science, Institute of Software, Chinese Academy of Sciences; University of Chinese Academy of Sciences, Beijing, China; E-mail: \{fusang, Beihong\}@iscas.ac.cn. Z. Chang, Telecom SudParis, Institut Polytechnique de Paris, Evry, France; Email: zhaoxin.chang@telecom-sudparis.eu. J. Xiong, College of Information and Computer Sciences, University of Massachusetts Amherst, USA; E-mail: jxiong@cs.umass.edu. R. Zheng, Department of Computing and Software, McMaster University, Canada; E-mail: rzheng@mcmaster.ca. J. Ma, Beijing University of Posts and Telecommunications, China; E-mail: junqi_ma@bupt.edu.cn. K. Niu, Peking University, Beijing, China, E-mail: xjtunk@pku.edu.cn. D. Zhang, Telecom SudParis, Institut Polytechnique de Paris, Evry, France, Key Laboratory of High Confidence Software Technologies (Ministryof Education), School of Electronics Engineering and Computer Science, Peking University, Beijing, China, E-mail: dqzhang@sei.pku.edu.cn. Corresponding Author: Daqing Zhang, Beihong Jin; E-mail: dqzhang@sei.pku.edu.cn, Beihong@iscas.ac.cn.

Permission to make digital or hard copies of all or part of this work for personal or classroom use is granted without fee provided that copies are not made or distributed for profit or commercial advantage and that copies bear this notice and the full citation on the first page. Copyrights for components of this work owned by others than ACM must be honored. Abstracting with credit is permitted. To copy otherwise, or republish, to post on servers or to redistribute to lists, requires prior specific permission and/or a fee. Request permissions from permissions@acm.org.

(C) 2021 Association for Computing Machinery.

2474-9567/2021/6-ART85 \$15.00

https://doi.org/10.1145/3463526

Proc. ACM Interact. Mob. Wearable Ubiquitous Technol., Vol. 5, No. 2, Article 85. Publication date: June 2021. 
Additional Key Words and Phrases: Contactless sensing; Long range sensing; LoRa Beamforming; Multi-target respiration sensing.

\section{ACM Reference Format:}

Fusang Zhang, Zhaoxin Chang, Jie Xiong, Rong Zheng, Junqi Ma, Kai Niu, Beihong Jin, and Daqing Zhang. 2021. Unlocking the Beamforming Potential of LoRa for Long-range Multi-target Respiration Sensing. Proc. ACM Interact. Mob. Wearable Ubiquitous Technol. 5, 2, Article 85 (June 2021), 25 pages. https://doi.org/10.1145/3463526

\section{INTRODUCTION}

In recent years, wireless sensing has attracted lots of attention from both academia and industry. Various wireless technologies traditionally mainly used for data communication, have now been exploited for contactfree wireless sensing. These wireless technologies include WiFi [1][2], RFID [3][4], 60GHz [5][6], UWB [7][8], ultrasound [9][10] and even visible light [11][12]. A large variety of new applications have been enabled by contact-free sensing ranging from fine-grained respiration monitoring [2], finger tracking [16] to coarse-grained fall detection [17], gait sensing [18] and daily activity recognition [19]. Though promising, one critical limit still remains: wireless sensing intrinsically has difficulties working with multiple targets. This is because wireless sensing relies on reflection signals from the subject to obtain the subject information such as movement direction and speed. When there exist multiple subjects, the reflections from multiple subjects get mixed at the receiver and the superimposed signal can not be utilized to sense any single subject. This limitation constrains wireless sensing to work with only single subject, preventing it from being used in most real-life scenarios with multiple subjects. For instance, to assess the sleep quality of a human subject, the vital signs (e.g., respiration) need to be continuously monitored to detect abnormal events such as sleep apnea. However, when there is a couple (Figure 1), obtaining the information from one of them becomes challenging because the signal reflected from the other individual can severely interfere. Sensing multiple targets is therefore a well-known challenge in contract-free wireless sensing.

Recently, researchers propose to employ LoRa signals to address another critical issue in wireless sensing: a small sensing range. This is because wireless sensing relies on reflection signal for sensing and the reflection signal is much weaker compared to the direct path signal. Therefore, even though the communication range of WiFi can reach tens of meters, the sensing range is just 6-8 meters [30]. The sensing range of acoustic signals is even smaller $(1-2 \mathrm{~m})$ [10]. The small sensing range further imposes restrictions on wireless sensing: the sensing target and the sensing device need to be in the same room and the target needs to be close to the sensing device in order to be sensed. These restrictions can not always be satisfied in reality. For example, the WiFi router may be in the living room and in this case, it is challenging to monitor a target in the bedroom. LoRa, on the other hand, provides us new opportunities to address this limited sensing range issue. LoRa is originally designed for long-range communication among IoT devices. The communication range is orders of magnitude higher than WiFi. In rural area, the transmission range can be up to a few kilometers [14]. The key to enable this long-range transmission is the Chirp Spread Spectrum (CSS) modulation and its high coding gain. Compared to WiFi which requires a 0-5 $d B$ SNR for decoding, LoRa signal can still be decoded even when the SNR is as low as $-20 d B$ [20,21]. Therefore, recently researchers propose to employ LoRa signals to significantly increase the sensing range from the state-of-the-art 6-8 $\mathrm{m}$ to 25-50 $\mathrm{m}$ [46][48]. While this is an applaudable step towards more practical wireless sensing, the interference issue becomes even more severe for LoRa sensing because a larger sensing range also means a larger interference range.

To address the interference issue, the straightforward solution is to separate signals reflected from multiple subjects and then sense each individual. Due to the narrow frequency bandwidth (i.e., $125 \mathrm{kHz}$ ) at LoRa, it is not possible to separate signals in time domain due to the low sampling rate. On the other hand, multiple antennas do exist on commodity LoRa gateway [15][32]. Therefore, in this work, we propose to employ multiple antennas 


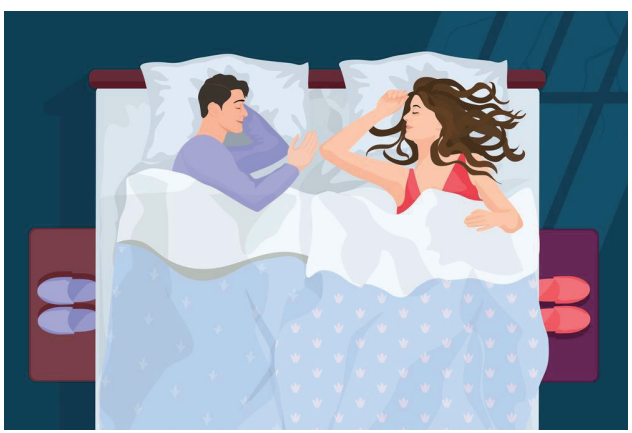

Fig. 1. Sleep monitoring of a couple.

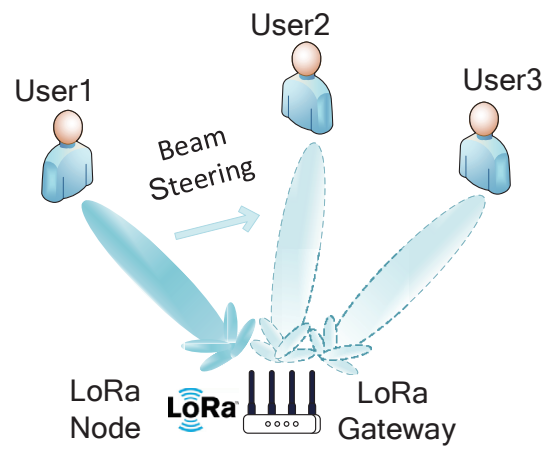

Fig. 2. LoRa beamforming for multi-target sensing.

to separate the signals in space domain to realize multi-target sensing. As illustrated in Figure 2, the basic idea of multi-antenna based beamforming is to strengthen signal reception at a specific direction so signals from other directions are much weaker which do not interfere the sensing at the specific direction.

However, we quickly found multiple challenges need to be tackled before this idea can be applied to enable multi-target sensing with LoRa.

- The first challenge is that traditional beamforming methods can not be applied on LoRa. To enable beamforming, either the transmitter-receiver pair needs to be synchronized (e.g., radar) or the channel information needs to be obtained (e.g., 802.11 ac WiFi). These two conditions can not be met for LoRa. The transmitter and receiver are spatially separated. Also, because the LoRa technology does not need to support high data rate like WiFi, the channel information which is used for MIMO transmission at WiFi is also not available with LoRa.

- The second challenge is that although beamforming helps to separate signals, the beamforming process can corrupt the signal amplitude and phase information which are the key parameters for wireless sensing.

- The third challenge for multi-target sensing is that traditional respiration sensing methods are locationdependent. When there are multiple targets, there is a high chance that some of the targets experience poor performance and even are undetected.

To tackle the aforementioned challenges, we develop a new LoRa beamforming technique which does not require transmitter-receiver synchronization or channel information. What is more important is that through careful signal processing, we make sure the beamforming procedure does not corrupt the signal amplitude/phase variation which is the key for sensing.

Specifically, to enable beamforming using unsynchronized LoRa transmitter-receiver, we need to deal with the carrier frequency offsets (CFO) and sampling frequency offsets (SFO). To eliminate the effects of these offsets, we propose a novel signal processing technique by constructing a "beam nulling" signal as a reference. By dividing the beamformed signal with the reference signal, we can successfully eliminate the unwanted phase offsets without corrupting the sensing information.

To address the location-dependent issue of traditional respiration sensing methods, we propose to exploit the dynamic path signal rather than the composite signal for sensing. Novel signal processing methods are proposed to obtain the location-independent chest displacement information for respiration sensing.

To validate the proposed ideas, we implement a real-time multi-target respiration monitoring system using a commodity LoRa node and a USRP based LoRa gateway. Extensive experiments conducted in different indoor environments show that the proposed system can detect and estimate the respiration rates of multiple human 
subjects in close proximity with higher than $96 \%$ accuracy even at a distance of $24 \mathrm{~m}$. A demo video can be found at https://youtu.be/_4RkDr3ZOyg.

The main contributions of this work are summarized as below:

- It enables long-range multi-target respiration sensing using LoRa signals.

- We propose the "beam nulling" method to address the unsynchronization issue between LoRa transmitter and receiver to enable beamforming.

- Different from traditional respiration sensing methods which employ the received composite signal for sensing, we propose novel signal processing method to exploit the dynamic component signal to address the location-dependent issue in respiration sensing.

- We prototype our design which can support multi-target respiration sensing in real time. Comprehensive experiments demonstrate its superior performance in terms of accuracy, sensing range and through-wall penetration capability compared with existing WiFi, mmWave radar and IR-UWB based solutions.

\section{BACKGROUND KNOWLEDGE}

\subsection{LoRa Primer}

LoRa adopts Chirp Spread Spectrum (CSS) modulation in the physical layer (PHY), which is tailored for long-range, low-power wireless communication. In LoRa, transmitted data is modulated as linear chirp signals. Given a certain carrier frequency denoted by $f_{c}$, a linear chirp's instantaneous frequency increases from $f_{c}-\frac{B}{2}$ to $f_{c}+\frac{B}{2}$ over $-\frac{T}{2} \leq t \leq \frac{T}{2}$, where $B$ is the bandwidth, and $T$ is the time duration of a chirp. The slope of a chirp, $k=\frac{B}{T}$, captures the rate of frequency change. A transmitted chirp signal can thus be represented as a complex exponential [29]:

$$
S(t)=e^{j 2 \pi f_{c} t+j \pi k t^{2}} .
$$

\subsection{LoRa Signals in Contact-free Sensing}

In contact-free sensing, we consider a signal traveling from a LoRa node (i.e., transmitter) to a LoRa gateway (i.e., receiver). Suppose that the LoRa node generates a chirp signal $S_{T}(t)=e^{j 2 \pi f_{c} t+j \pi k t^{2}}$. The received signal consists of two components: a static component and a dynamic component. The static component, denoted by a vector $H_{s}$, is the result of signal propagation along the direct line-of-sight (LoS) path between the transmitter and receiver, and reflection paths from stationary objects in the environment. The dynamic component corresponds to reflection signals from moving targets. The superimposed received signal can thus be represented as:

$$
S_{R}(t)=e^{j \pi k t^{2}+j 2 \pi f_{c} t}\left(H_{S}+A e^{-j \frac{2 \pi d(t)}{\lambda}}\right) .
$$

where $A$ is the amplitude of dynamic path component, $d(t)$ is the length of the dynamic path, and $\lambda$ is the signal wavelength. The LoRa receiver demodulates the received signal by multiplying it with $e^{-j 2 \pi f_{c}^{\prime} t}$, where $f_{c}^{\prime}$ is the carrier frequency at the gateway. Note that ideally the carrier frequency at the transmitter should be the same as the carrier frequency at the receiver: $f_{c}^{\prime}=f_{c}$. In reality, there is always a small difference between $f_{c}^{\prime}$ and $f_{c}$ which is called Carrier Frequency Offset (CFO). The demodulated signal can then be represented as:

$$
R(t)=e^{j\left(\pi k t^{2}+\phi_{c}+\phi_{s}\right)}\left(H_{s}+A e^{-j \frac{2 \pi d(t)}{\lambda}}\right),
$$

where $e^{j\left(\pi k t^{2}+\phi_{c}+\phi_{s}\right)}$ is random phase offset caused by CFO and SFO. $\phi_{c}=2 \pi\left(f_{c}-f_{c}^{\prime}\right) t$ is the CFO. Due to unsynchronized clocks at LoRa transceivers, $\phi_{s}$ is the phase error introduced by Sampling Frequency Offset (SFO). $\mathrm{CFO}$ and $\mathrm{SFO}$ are caused by the asynchronous transmitter and receiver which is different from the case (transmitter and receiver are synchronized) in radar systems. 


\subsection{Beamforming}

Beamforming is a powerful technique which has been widely used in radar and modern communication systems (e.g., IEEE $802.11 \mathrm{ac}$ ). The basic idea is through signal processing, a stronger reception/transmission at the direction of interest is achieved. Beamforming techniques can be broadly divided into two categories: 1) mechanically switched beamforming and 2) phase-array based beamforming [13]. Phase-array based beamformers can tune the beamforming direction quickly by varying the signal amplitude and phase at each antenna element. Compared to mechanically switch beamforming, phase-array based beamformers can switch signal directions much faster.

\section{LORA BEAMFORMING}

In this section, we introduce the design of LoRa beamforming, which serves as the basis of multi-target sensing.

\subsection{Beamforming Design}

To illustrate the concept of beamforming at LoRa, we consider a LoRa gateway with a linear array of $M$ antennas. As shown in Figure 3, the chirp signal reflected from a human body experiences different amounts of propagation distances at different antennas. We denote the path lengths of the signal from the human subject to the $M$ antennas as $d_{1}(t), d_{2}(t), .$. and $d_{M}(t)$, respectively. Note that besides the dynamic signal reflected from the human subject, there are direct-path LoS signal between the transmitter-receiver pair and also reflection paths from static objects in the environment such as walls and furniture. We use a vector to represent the dynamic signal and another vector to represent the grouped static signal at each antenna element. The corresponding dynamic vectors and static vectors at the $M$ antenna elements are denoted as $H_{d 1}, H_{d 2}, \ldots, H_{d M}$ and $H_{s 1}, H_{s 2}, \ldots, H_{s M}$, respectively. At each antenna element, the static vector is a constant. The amplitude of the dynamic vector can also be considered as a constant for a small displacement (e.g., $5 \mathrm{~mm}$ ) induced by movements such as human respiration. On the other hand, the phase change is significant. For a displacement of $5 \mathrm{~mm}$, the phase change is $11^{\circ}$ which can not be ignored. Therefore, in the complex I-Q domain as shown in Figure 3, if we visualize the relationship between the static vector and dynamic vector, the dynamic vector rotates with respect to the static vector during the process of human respiration.

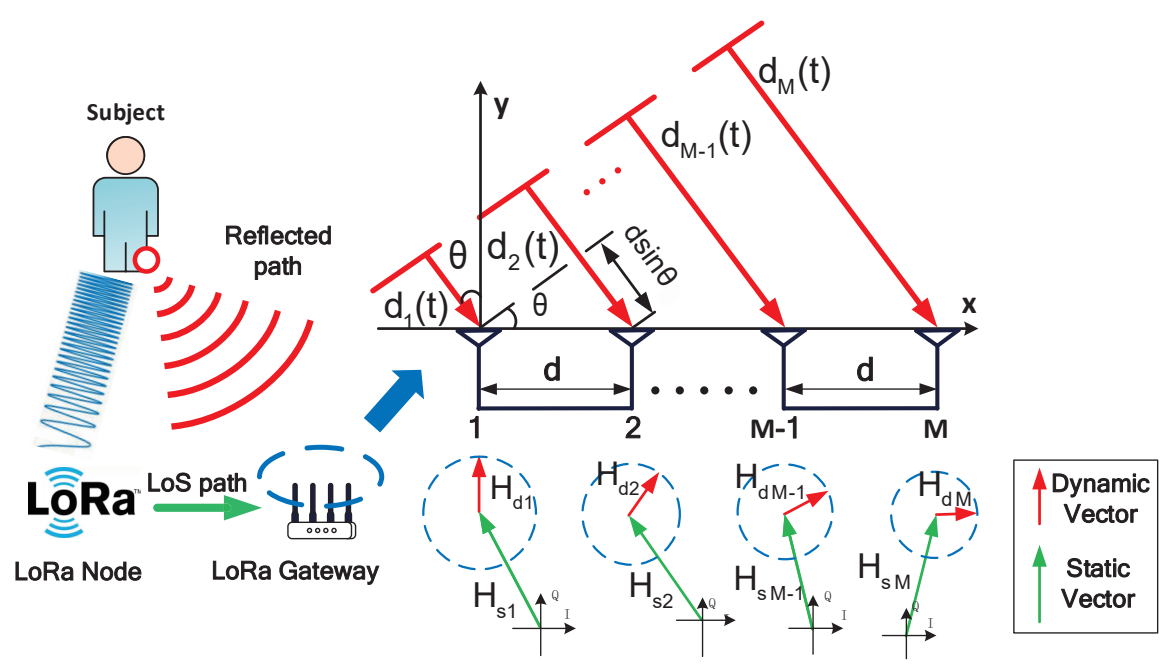

Fig. 3. Static and dynamic components at multiple LoRa gateway antennas. 
Because the signals received at multiple antennas are from a single source, the sweep parameters of these chirp signals are the same. From Eq. (3), the demodulated baseband signals obtained at the $M$ receiving antennas are given by:

$$
\begin{gathered}
R_{1}(t)=e^{j\left(\pi k t^{2}+\phi_{c}+\phi_{s}\right)}\left(H_{s 1}+A_{1} e^{-j \frac{2 \pi d_{1}(t)}{\lambda}}\right), \\
R_{2}(t)=e^{j\left(\pi k t^{2}+\phi_{c}+\phi_{s}\right)}\left(H_{s 2}+A_{2} e^{-j \frac{2 \pi d_{2}(t)}{\lambda}}\right), \\
\vdots \\
R_{M}(t)=e^{j\left(\pi k t^{2}+\phi_{c}+\phi_{s}\right)}\left(H_{s M}+A_{M} e^{-j \frac{2 \pi d_{M}(t)}{\lambda}}\right) .
\end{gathered}
$$

To achieve a stronger signal reception at a particular direction, we can sum up the delayed version of signals from multiple antennas. Suppose the linear antenna array is uniformly spaced with a spacing of $d$ between adjacent antennas. Note that for LoRa signal at $915 \mathrm{MHz}, d=16 \mathrm{~cm}$. On the other hand, the distance between the target and the antenna array can be tens of meters, which is much larger than $d$. Therefore, we can assume the angles from the target to each antenna are the same.

The signals received at the 2 nd antenna to $M$ th antenna are delayed versions of the signal received at the 1st antenna. Therefore, there is an extra amount of signal propagation in the air and accordingly an additional phase rotation at the $m$ th antenna with respect to the first antenna. When the signal arrival angle is $\theta$ as shown in Figure 3, the phase difference between the dynamic signals arriving at the $m$ th and the first antenna is given by:

$$
\Delta \phi_{m}(\theta)=(m-1) \cdot \frac{2 \pi}{\lambda} \cdot d \cdot \sin (\theta) .
$$

For an arbitrary signal arrival angle $\theta$, a steering vector is thus defined to represent the phase difference between the rest antennas and the first antenna as:

$$
\omega(\theta)=\left[e^{j \Delta \phi_{1}(\theta)}, e^{j \Delta \phi_{2}(\theta)}, \ldots, e^{j \Delta \phi_{M}(\theta)}\right] .
$$

Formally, by computing the weighted sum of the received signals at all $M$ antennas, we construct the beamformed signal received at direction $\theta$ as follows:

$$
\begin{aligned}
& y(\theta, t)=\sum_{i=1}^{M} \omega_{i}(\theta) \cdot R_{i}(t)=\sum_{i=1}^{M} e^{j \Delta \phi_{i}(\theta)} R_{i}(t) \\
& =\underbrace{\underbrace{j\left(\pi k t^{2}+\phi_{c}+\phi_{s}\right.})}_{\text {unwanted phase shift caused by CFO and SFO }} \cdot[\underbrace{\sum_{i=1}^{M} H_{s i} \cdot e^{j \Delta \phi_{i}(\theta)}}_{\text {Static vector combination }}+\underbrace{\left.\sum_{i=1}^{M} A_{i}(t) \cdot e^{-j \frac{2 \pi d_{i}(t)}{\lambda}} \cdot e^{j \Delta \phi_{i}(\theta)}\right]}_{\text {Dynamic vector combination }} .
\end{aligned}
$$

To simplify Eq. 7, we further denote static vector combination $\sum_{i=1}^{M} H_{s i} \cdot e^{j \Delta \phi_{i}(\theta)}$ as $H_{s}^{\text {beam }}$ which is a constant. The dynamic vector combination can be simplified as below:

$$
\sum_{i=1}^{M} A_{i}(t) \cdot e^{-j \frac{2 \pi d_{i}(t)}{\lambda}} \cdot e^{j \Delta \phi_{i}(\theta)}=\sum_{i=1}^{M} A_{i}(t) \cdot e^{-j \frac{2 \pi\left(d_{i}(t)-(i-1) d \cdot \sin (\theta)\right)}{\lambda}}=\sum_{i=1}^{M} A_{i}(t) e^{-j \frac{2 \pi d_{1}(t)}{\lambda}}
$$

Note that $d_{i}(t)-(i-1) d \sin (\theta)$ exactly equals to $d_{1}(t)$, which means the phases of signal at the $2 n d$ antenna to $M t h$ antenna are aligned with the first antenna. Let $A^{\text {beam }}=\sum_{i=1}^{M} A_{i}(t)$ be the energy sum of the dynamic components at all $M$ antennas. Eq. 7 can thus be simplified as:

$$
y(\theta, t)=e^{j\left(\pi k t^{2}+\phi_{c}+\phi_{s}\right)}\left(H_{s}^{\text {beam }}+A^{\text {beam }} e^{-j \frac{2 \pi d_{1}(t)}{\lambda}}\right) .
$$


From Figure 4 and Eq. (9), we know that the beamformed signal consists of both static and dynamic components. By choosing the right $\theta$, the reflection signals (dynamic components) from the target at $M$ antennas add up constructively while those (static components) randomly add up.

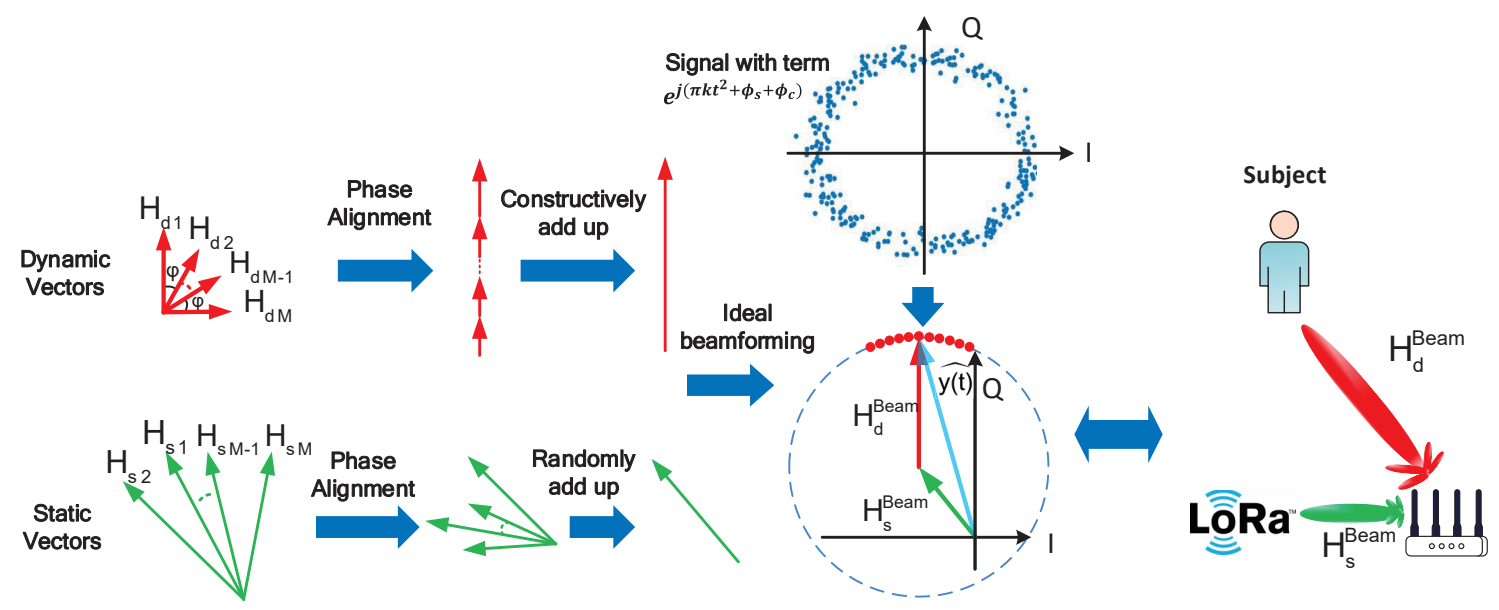

Fig. 4. Composition of static and dynamic vectors from multiple antennas.

Figure 4 provides an illustrative example of this process. We can see that after beamforming, dynamic vector (red arrow) containing the target information is greatly enhanced (due to constructively added up) while the amplitude of the static vector does not change much (due to randomly added up). Therefore, in the vector space, now the dynamic vector rotates with respect to the static vector, inducing a larger signal variation compared to that before beamforming. However, this large variation still can not present us clear target information because the phase and amplitude changes are corrupted by the $\mathrm{CFO}$ and $\mathrm{SFO}$ which are time varying.

We can view the resulted signal as a dynamic vector rotating around a static vector in the $\mathrm{I} / \mathrm{Q}$ complex domain. To eliminate the undesired random phase rotation caused by $\mathrm{CFO}$ and $\mathrm{SFO}$, we need to remove the term $e^{j\left(\pi k t^{2}+\phi_{c}+\phi_{s}\right)}$ in Eq. (7). Note that for radar system with synchronized transmitter and receiver, CFO and SFO are both 0 . However, the LoRa transmitter and receiver are spatially separated and unsynchronized, and the receiver does not know the transmitted signal. Thus, traditional beamforming method does not work with LoRa. Existing LoRa sensing work [46] calculates the ratio of two signals obtained from two antennas at the receiver to eliminate the random phase offsets. However, during this process, the sensing information (signal phase and amplitude) are corrupted because the signal received at each antenna always contains the time-varying dynamic component. Therefore, we need to find another reference signal without the dynamic component to eliminate the random phase offset and at the same time still preserve the information for sensing.

Next, we explain how to accomplish this. Our objective is to find a reference signal that can cancel the unwanted part containing the random phase offsets (CFO and SFO). We also need to make sure during the process, the dynamic component which contains the target sensing information is not affected. To achieve this, we perform the opposite operation of the beamforming process to destructively combine the dynamic component received at multiple antennas. We term this process "beam nulling". Let $W=\left[r_{1} e^{j \varepsilon_{1}}, r_{2} e^{j \varepsilon_{2}}, \ldots, r_{M} e^{j \varepsilon_{M}}\right]$ be a $1 \times M$ weight vector with $2 M$ parameters to be determined. We multiply the signal received at each antenna with the weight 
and sum them together. The weighted sum of signals at these antennas is thus given by:

$$
\begin{aligned}
& \sum_{i=1}^{M} W_{i} \cdot R_{i}(t)=\sum_{i=1}^{M} r_{i} e^{j \varepsilon_{i}} R_{i}(t) \\
& =\underbrace{e^{j\left(\pi k t^{2}+\phi_{c}+\phi_{s}\right)}}_{\text {Unwanted phase rotation caused by } \mathrm{CFO} \text { and SFO }} \cdot[\underbrace{\sum_{i=1}^{M} H_{s i} \cdot r_{i} e^{j \varepsilon_{i}}}+\underbrace{\sum_{i=1}^{M} A_{i}(t) \cdot e^{-j \frac{2 \pi d_{i}(t)}{\lambda}} \cdot r_{i} e^{j \varepsilon_{i}}}]^{.} \\
& \text {Static vector combination Dynamic vector combination }
\end{aligned}
$$

As shown in Figure 5, to null out the dynamic component, one needs to find a $1 \times M$ weight vector $W$ such that:

$$
\sum_{i=1}^{M} A_{i}(t) \cdot e^{-j \frac{2 \pi d_{i}(t)}{\lambda}} \cdot r_{i} e^{j \varepsilon_{i}}=0
$$

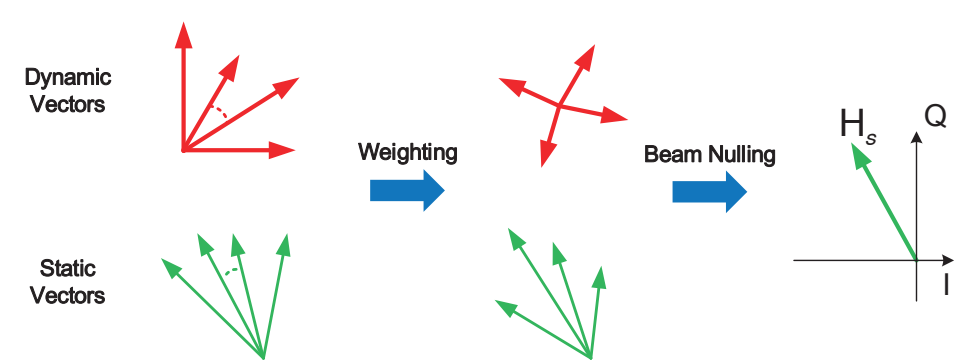

Fig. 5. Beam nulling: null out dynamic vectors by a weighted sum of different antennas.

However, since we have no priori knowledge about the magnitude of the dynamic vector at each antenna, it is not possible to obtain the weight solution $W$ by directly solving Eq. (11). Instead, we formulate it as an optimizing problem. Specifically, given $W$, we define the corresponding energy ratio of the dynamic frequency components to the total signal energy as follows:

Definition 3.1. Energy ratio of the dynamic frequency component is defined as the ratio of subject motion ${ }^{1}$ energy to the overall energy in the frequency spectrum. Specifically, the calculation of energy ratio consists of two steps: 1) the dynamic frequency component is obtained by taking Fast Fourier Transform (FFT) of the weighted sum signal over a time window; 2) the energy sum of bin containing the FFT peak and its adjacent bins divide by the energy sum of all FFT bins.

To find the optimal $W$, we apply the stochastic optimization algorithm [55] to iteratively refine the search. Let $W^{*}$ be the resulted solution and the resulted beam-nulled signal from Eq. 12 is:

$$
R e f(t)=\sum_{i=1}^{M} W_{i}^{*} \cdot R_{i}(t)=\sum_{i=1}^{M} r_{i}^{*} e^{j \varepsilon_{i}^{*}} R_{i}(t)=e^{j\left(\pi k t^{2}+\phi_{c}+\phi_{s}\right)} \cdot \sum_{i=1}^{M} H_{s i} \cdot r_{i}^{*} e^{j \varepsilon_{i}^{*}} .
$$

Thus we can quickly obtain the "beam null" reference signal containing only the static component and the unwanted part. We denote this signal as $\operatorname{Re} f(t)=e^{j\left(\pi k t^{2}+\phi_{c}+\phi_{s}\right)} \cdot H_{s}^{R e f}$ and $H_{s}^{R e f}=\sum_{i=1}^{M} H_{s i} \cdot r_{i}^{*} e^{j \varepsilon_{i}^{*}}$.

Now, to cancel out the unwanted part, we divide $y(\theta, t)$ by $\operatorname{Re} f(t)$ using Eq. 9 and Eq. 12 as follows:

$$
\hat{y}(\theta, t)=\frac{y(\theta, t)}{\operatorname{Ref}(t)}=\frac{\overline{e^{\pi k t^{2}+\phi_{c}+\phi_{s}}}\left(H_{s}^{\text {beam }}+A^{\text {beam }} e^{-j \frac{2 \pi d_{1}(t)}{\lambda}}\right)}{e^{\pi k t^{2}+\phi_{c}+\phi_{s}} \cdot H_{s}^{R e f}}=\overline{H_{s}^{\text {beam }}}+\overline{A^{\text {beam }}} e^{-j \frac{2 \pi d_{1}(t)}{\lambda}},
$$

\footnotetext{
${ }^{1}$ If the subject motion is human respiration, we set the frequency range (i.e., 0.1-0.5 Hz) of normal respiration rate (10-30 BPM).
} 
where $\widehat{H_{s}^{\text {beam }}}=\frac{H_{s}^{\text {beam }}}{H_{s}^{\text {Ref }}}$ is the estimated constant static vector and $\widehat{A^{\text {beam }}}=\frac{A^{\text {beam }}}{H_{s}^{\text {Ref }}}$ is the enhanced beamformed dynamic vector.

From Eq. (13), we can see that the varying random phase offsets are eliminated. The remaining part only depends on the dynamic path length $d_{1}(t)$ and some constants (i.e., the static vector). The dynamic component gets strengthened without corrupting the sensing information.

\subsection{Understanding the Effects of LoRa Beamforming}

Next, we employ LoRa beamforming to sense movements of multiple targets and present the results from our benchmark experiments.

First, we simulate the beam patterns by steering the beam at different directions. In the simulation, the gateway is configured with 4 antennas, and the beam steering direction is set to $60^{\circ}, 30^{\circ}, 0^{\circ},-30^{\circ}$ and $-60^{\circ}$. As shown in Figure 6, with phase compensation and normalization, the beam is effectively pointed at the specified directions. We can observe that the sidelobes of the beam pattern for larger angles (60 and -60) are more pronounced than those at small angles. However, the main lobe still points quite accurately towards the targeted direction. If the number of antennas increases, the beam width is thinner, resulting in a higher spatial resolution.
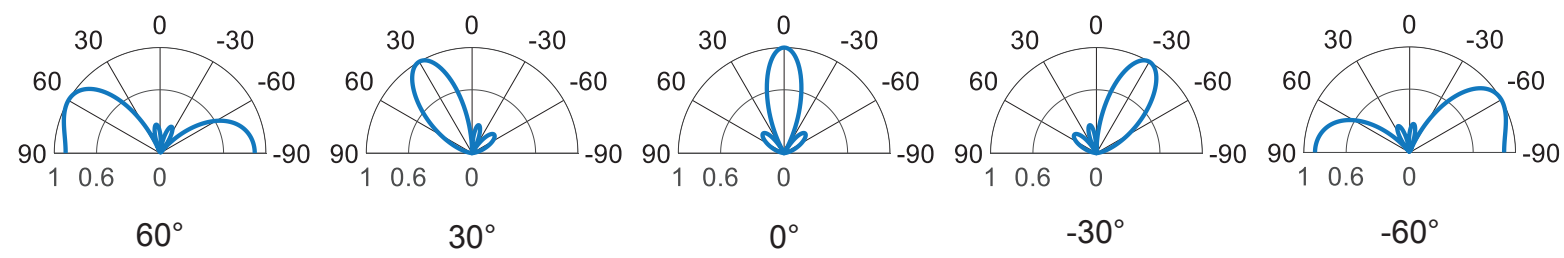

Fig. 6. Effects of beamforming.

Next, we carry out beachmark experiments to validate the proposed beamforming technique for sensing the movements of multiple targets. In Figure 7a, we place objects at five different locations in an indoor environment. These locations are at different beam steering angles with respect to the LoRa device, ranging from $60^{\circ}$ to $-60^{\circ}$ at a step size of $30^{\circ}$. The objects (i.e., metal cylinders as shown in Figure 7b) move along a sliding track of $1 \mathrm{~m}$ forward and backward controlled by a Raspberry Pi 3 Model B. Five different speed levels are considered including very fast $(12 \mathrm{~cm} / \mathrm{s})$, fast $(6 \mathrm{~cm} / \mathrm{s})$, medium $(3 \mathrm{~cm} / \mathrm{s})$, slow $(1.5 \mathrm{~cm} / \mathrm{s})$ and super slow $(0.7 \mathrm{~cm} / \mathrm{s})$.

In this experiment, we would like to study if the information of each target can be clearly obtained without being interfered by applying the proposed beamforming method. Note that, if we do not apply the proposed beamforming technique, signal reflections from multiple targets are mixed and it is challenging to sense any of the targets.

Figure 8 shows the normalized amplitudes of the signals from each target. When there are three objects moving at speed of $6 \mathrm{~cm} / \mathrm{s}, 12 \mathrm{~cm} / \mathrm{s}$ and $3 \mathrm{~cm} / \mathrm{s}$ at locations $P 2, P 3$ and $P 4$ respectively (Figure $8 \mathrm{a}$ ), we observe the beamformed signals at the direction of $P 2, P 3$ and $P 4$ exhibit clear cyclic patterns, corresponding to the target movements. Note that the speed information of each target is also accurately captured. We can observe 11, 22 and 5.5 cycles over a 30 -second interval corresponding to three different speeds $(6 \mathrm{~cm} / \mathrm{s}, 12 \mathrm{~cm} / \mathrm{s}$ and $3 \mathrm{~cm} / \mathrm{s})$. Note that for a $6 \mathrm{~cm} / \mathrm{s}$ of speed, $30 \mathrm{~s}$ means a movement of $\Delta d=180 \mathrm{~cm}$. The theoretical number of signal variation cycles is $2 \Delta d / \lambda=360 \mathrm{~cm} / 32.79 \mathrm{~cm}=11$. We can see that the experiment results perfectly match the theoretical analysis.

The signal variations at other directions (i.e., $P 1$ and $P 5$ ) are much weaker because there are no target reflections from those directions. The average signal variance from a direction with no object is less than $20 \%$ of that in the 


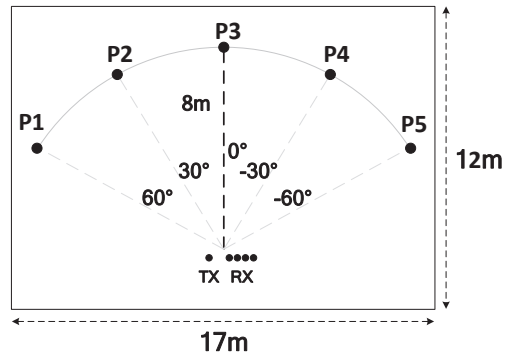

(a) Experiment environment

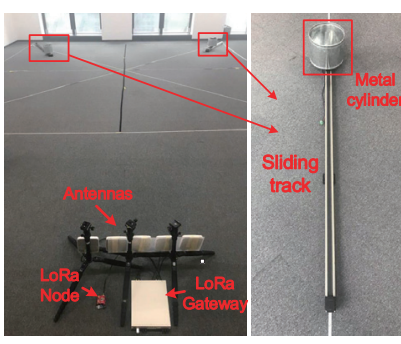

(b) LoRa devices and equipments

Fig. 7. LoRa beamforming validation.
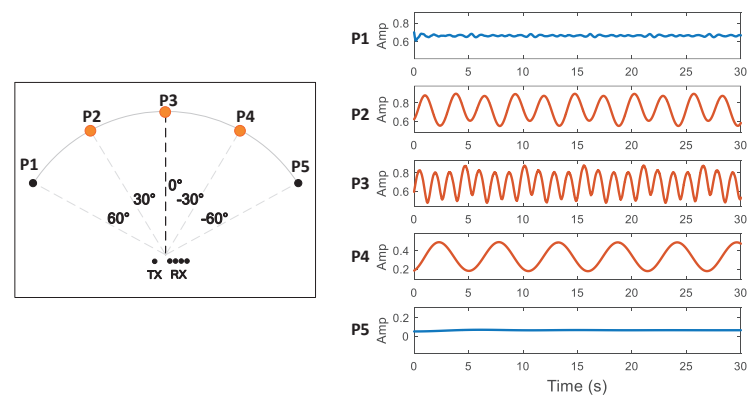

(a) Three objects at P2, P3 \& P4
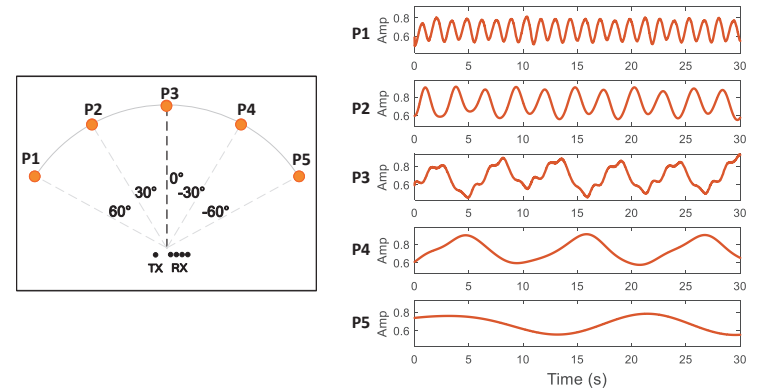

(b) Five objects at P1-P5

Fig. 8. LoRa beamforming validation.

direction with a moving object. Similar observations can be made with five objects in Figure 8b. Furthermore, from the figures, different speeds can be clearly identified from the frequencies of the waveforms. These experiments demonstrate the promises of the proposed beamforming technique and signal representation scheme in separating moving targets in different directions and estimating their respective moving speed.

\section{MULTI-TARGET RESPIRATION SENSING}

In this section, we build upon the proposed LoRa beamforming technique to develop novel methods for finegrained multi-target respiration sensing. First, we illustrate how subtle chest displacements induced by human respiration affect LoRa signals, and discuss the challenges in estimating respiration rates. Next, we introduce the location-dependent issue when employing the composite signal for respiration sensing and present our method to exploit the dynamic signal to achieve location-independent sensing. We also show how the proposed method can be applied to distinguish subjects with similar respiration rates which is also a challenge in multi-target respiration sensing.

\subsection{Dynamic Vector vs. Composite Vector for Sensing}

According to the previous study [22], chest displacements during respiration are about $1 \mathrm{~mm}-12 \mathrm{~mm}$. The inhalation and exhalation of chest motions cause path length change of the reflected LoRa signals, denoted by $\Delta d$. We convert such path length change to corresponding rotation of the dynamic vector in the complex I-Q 
plane. The rotation angle $\phi$ caused by respiration is calculated as $\phi=2 \pi \times \frac{2 \Delta d}{\lambda}$. $\lambda$ is the wavelength of the LoRa signals, which is $32.79 \mathrm{~cm}$ for $915 \mathrm{MHz}$ carrier frequency. Therefore, the dynamic vector rotates $2.2^{\circ}-26^{\circ}$ for a chest displacement of $1 \mathrm{~mm}-12 \mathrm{~mm}$.

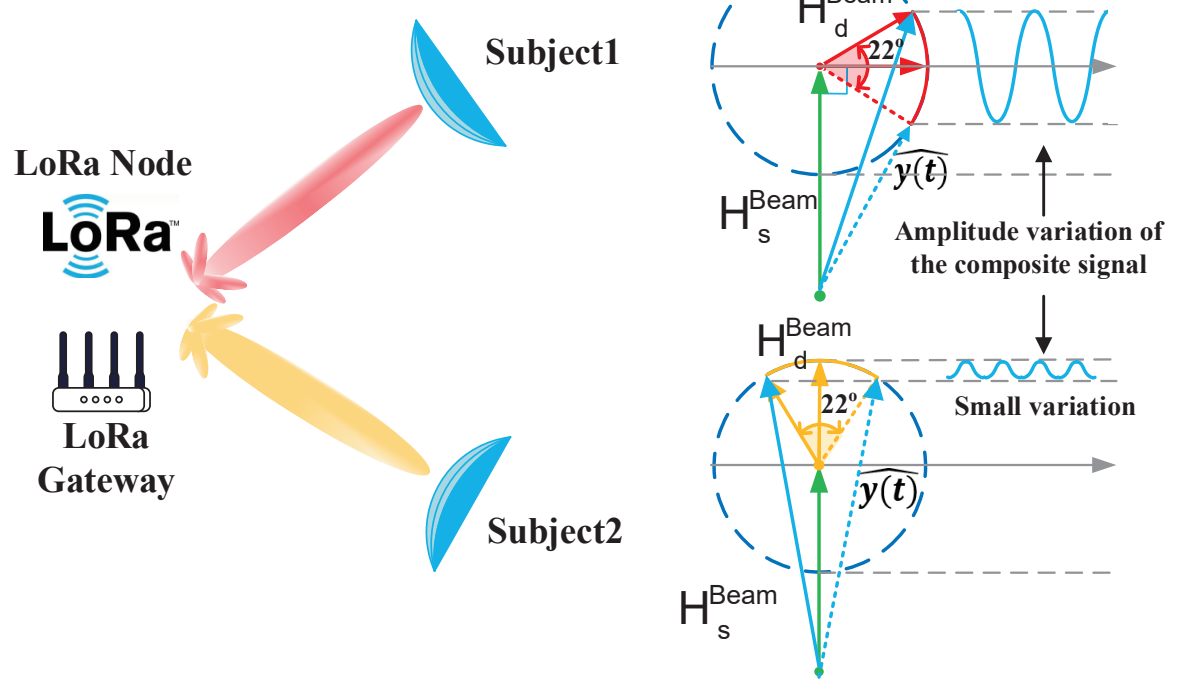

Fig. 9. Sensing two targets' respiration at different beam directions.

Now we consider two subjects breathing naturally at two different locations. For a chest displacement of $1 \mathrm{~cm}$, the dynamic vector rotates $22^{\circ}$, as illustrated by the red and yellow arcs in Figure 9. However, note that the signal retrieved from the receiver is the composite signal which is a superposition of the static and dynamic signals. As the static signal component is unknown, the dynamic signal component can not be obtained. Therefore, existing sensing systems exploit the composite signal for sensing. However, one critical issue exists and we illustrate the issue below.

Let us assume the chest displacements of the two subjects are the same $(1 \mathrm{~cm})$. As shown in Figure 9, the red arc and yellow arc are of the same length. The blue color arrows indicate the composite signals and the blue color waves represent the amplitude change of the composite signals. Some interesting observation can be obtained: the same amount of dynamic component rotation causes dramatically different amounts of composite signal variations when the subjects are located at different locations. This brings in one severe issue: the sensing performance is location dependent and therefore is unstable. When the target is located at some "bad" locations, the sensing performance is poor. Poor performance corresponds to a smaller composite signal variation which can easily be buried in noise without being detected. When there are multiple targets, if we employ the composite signal variation for sensing, some targets at "bad" locations can be undetected. Therefore, in this paper, we propose to exploit the dynamic signal component for sensing. This is a non-trivial task and we present our novel solution in the next section.

\subsection{Exploiting the Dynamic Signal Component for Sensing}

From the previous section, we know that when the composite signal is used for sensing, the sensing performance is location dependent. To address this issue, the straightforward solution is to remove the static signal component and only keep the dynamic signal component. The variation of the dynamic component is location independent. 
To remove the static component, existing system [52] estimates the static component by averaging the signal samples collected for a full cycle (the dynamic vector rotates for $360^{\circ}$ ). This method works because the dynamic components collected during a full cycle can cancel each other. The static component can thus be obtained and removed from the composite vector to obtain the dynamic vector. However, this method requires a subject to move a relatively large distance in order for the dynamic vector to rotate for 360 degrees. In the case of respiration with just subtle chest displacements, the amount of dynamic vector rotation is much less than $360^{\circ}\left(22^{\circ}\right.$ for a chest displacement of $1 \mathrm{~mm}$ ). Therefore, for respiration, the static component cannot be estimated using this method.

In this paper, we propose a new method to deal with the location-dependent issue of the composite signal when used for sensing. The key observation to make this happen is that for respiration sensing, the two key parameters, respiration rate and respiration depth (chest displacement) can be obtained from the phase rotation of the dynamic vector. We propose to calculate the phase rotation of the dynamic vector using just the composite vectors available at the receiver. The key concept is illustrated as below. As shown in Figure 10, the dynamic vector rotates during the process of respiration. The amount of rotation can be used to determine the chest displacement. As shown in previous section, a chest displacement of $1 \mathrm{~mm}$ corresponds to a 22 degree of dynamic vector rotation. Inversely, if we know the amount of dynamic vector rotation, we can calculate the amount of chest displacement. The time interval taken for the dynamic vector to rotate corresponds to the respiration rate. A smaller time interval indicates a faster respiration rate.

Note that if we know the vectors for 3 adjacent points on the arc (e.g., A, B, C as shown in Figure10) of the dynamic vector, we can calculate the curvature of the circle and fully characterize the rotation of the dynamic vector. However, at the receiver, the dynamic vector is not available and only composite signal (vector) can be obtained. Interestingly, as the static vector is a constant, we can use the three corresponding composite vectors ( $\mathrm{A}^{\prime}$, $B^{\prime}$ and $C^{\prime}$ as shown in Figure 10) to calculate the curvature of the arc, equivalent to using the three dynamic vectors (A, B and C).

This curvature information presents us two more unique advantages for multi-target sensing. As shown in Figure 12, we present the curvature plots for two subjects with similar respiration rate. The first unique advantage is that we can use the large changing point to detect a target. As the curvature value of the changing point is very large, we can achieve very robust performance for target detection. The second unique advantage is that the curvature curve can be used to differentiate two targets. Even they have the same respiration rate, it is very unlikely for their respiration process to be time-synchronized (the changing points occur exactly at the

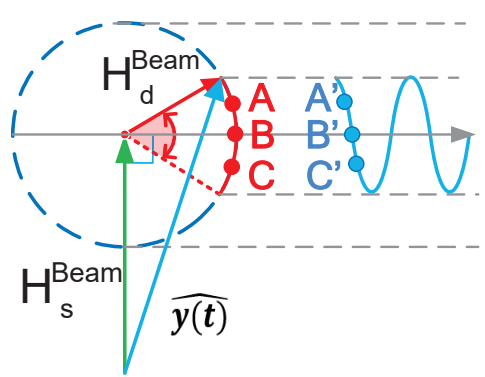

Fig. 10. Points on dynamic vector vs. composite vector.

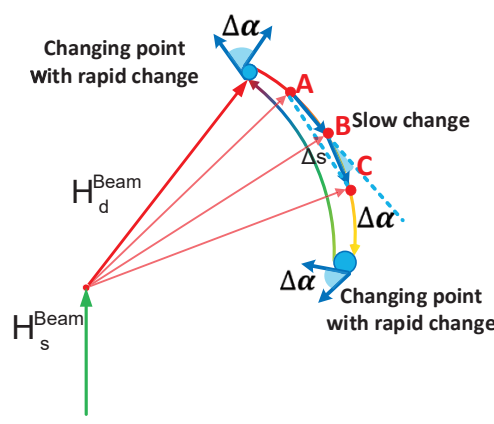

Fig. 11. Curvature calculation.

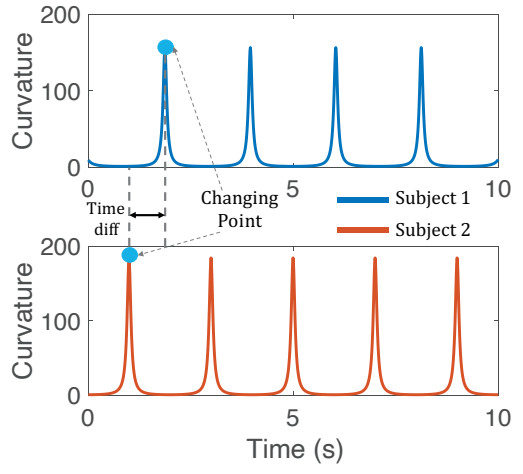

Fig. 12. Curvature of arc in time domain. 
same time) in time domain. Therefore, we can use the curvature curve to differentiate targets even with similar respiration rates.

Now, let us illustrate how to calculate the curvature using three adjacent vector points on the arc. The curvature of the arc at point $p$ is the rate of change at the tangential direction. As an example, consider three points $A, B, C$ in Figure 11. Let $\Delta \alpha=\operatorname{Angle}(\overrightarrow{A B})-\operatorname{Angle}(\overrightarrow{B C})$ be the change of tangential angles between $A$ and $C$ and $\Delta s$ is the length of arc $A C$. The curvature $\kappa$ at $C$ is thus computed by moving $A$ and $C$ infinitely closer to $B$ as:

$$
\kappa=\lim _{\Delta s \rightarrow 0}\left|\frac{\operatorname{Angle}(\overrightarrow{A B})-\operatorname{Angle}(\overrightarrow{B C})}{\Delta s}\right|=\lim _{\Delta s \rightarrow 0}\left|\frac{\Delta \alpha}{\Delta s}\right|
$$

Figure 12 shows the estimated curvature curves over time associated with chest motions during respiration. The peak curvatures indicate the transition between exhalation and inhalation. Most importantly, the curvature information is location independent and is only related to the characteristic of the respiration process.

\subsection{Multi-target Detection and Respiration Rate Estimation}

Figure 13 illustrates the complete pipeline of the proposed multi-target detection and respiration rate estimation process. It consists of three core modules: (1) beamformed signal construction, (2) direction-frequency spectrogram pre-processing, and (3) multi-target detection and respiration rate estimation. Next, we present the details of each module.

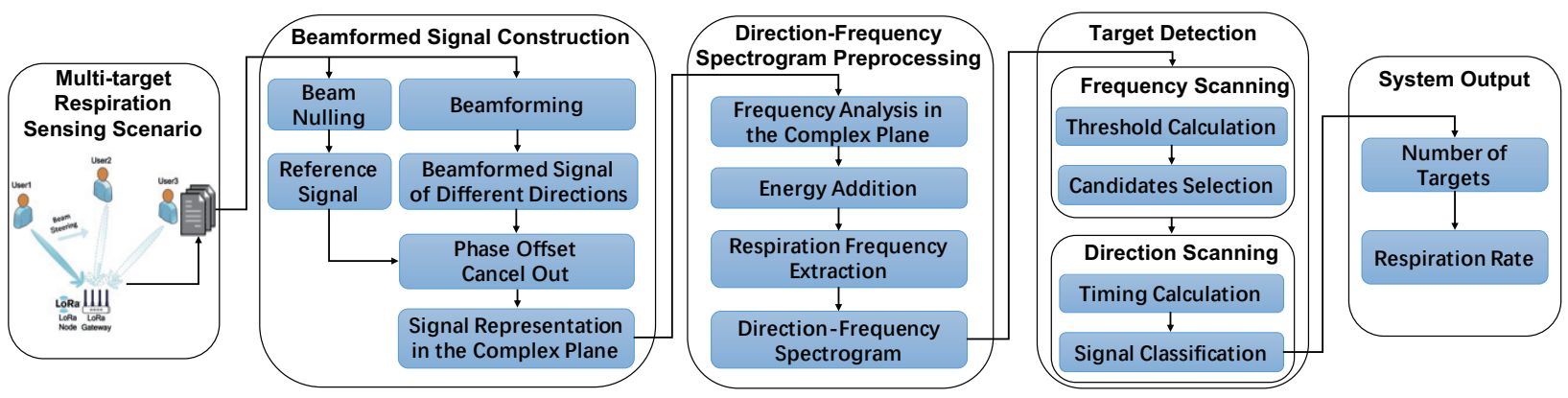

Fig. 13. The process of multi-target detection and respiration rate estimation.

4.3.1 Beamformed Signal Construction. First, we scan all directions between $-80^{\circ}$ and $80^{\circ}$ (at a step size of $\left.1^{\circ}\right)^{2}$ To scan each direction, the raw signal vector is multiplied by the corresponding steering vector to strengthen the signal at this direction. Since the phases of these beamformed signals still contain phase offsets caused by CFO and SFO, we apply the "beam nulling" scheme presented in Section 3.1 to construct the reference signal. Next, we divide the beamformed signal by the reference signal to cancel out the random phase offsets. The resulted representation is a complex signal.

4.3.2 Direction-Frequency Spectrogram Pre-processing. To pre-process the beamformed signals, we generate a Direction-Frequency spectrogram. Firstly, we apply the Fast Fourier Transform (FFT) to the beamformed signals in the complex plane in each direction. Then a band-pass filter in the range of human respiration rate $(0.1-0.5 \mathrm{~Hz})$ is applied. The resulted Direction-Frequency spectrogram thus characterizes the distribution of respiration signal energy at various directions. An example can be found in Figure 14, where four people breathe naturally in one

${ }^{2}$ The speed of digital scanning is very fast. According to our experiment, it only takes $1.25 \mathrm{~ms}$ to scan one direction and $200 \mathrm{~ms}$ to scan the whole range of $160^{\circ}$. 


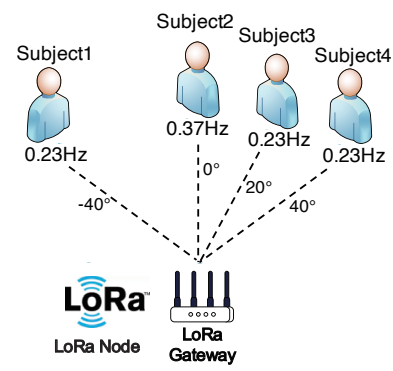

Fig. 14. Scenario of four targets respiration sensing.

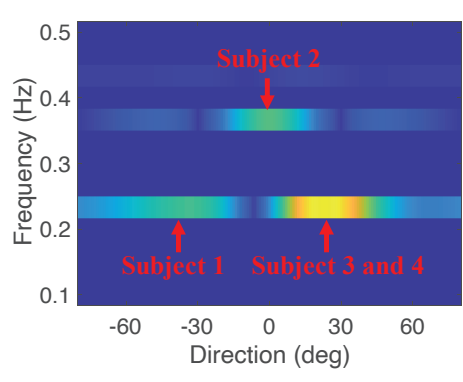

Fig. 15. Direction-Frequency Spectrogram.

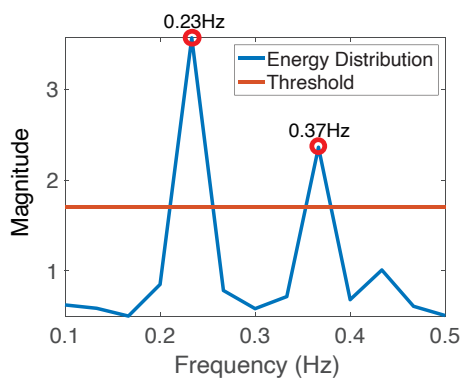

Fig. 16. Candidate frequencies selection.

room. Their location directions are $-40^{\circ}, 0^{\circ}, 20^{\circ}$ and $40^{\circ}$ with respect to the LoRa devices, and their respective respiration rates are $0.23 \mathrm{~Hz}, 0.37 \mathrm{~Hz}, 0.23 \mathrm{~Hz}, 0.23 \mathrm{~Hz}$. From the generated direction-frequency spectrogram in Figure 15, one can clearly observe three bright clusters. The first subject with a $0.23 \mathrm{~Hz}$ respiration rate sits at $-40^{\circ}$, and the second subject with a $0.37 \mathrm{~Hz}$ respiration rate sits at $0^{\circ}$. Since subject 3 and 4 have the same respiration rate and are at close directions, they are not distinguishable in the spectrogram. We will present how to distinguish them below.

4.3.3 Multi-target Detection. In the direction-frequency spectrogram, each subject is associated with two features: direction and frequency. However, subject detection still faces a problem. Due to the existence of beamforming side lobes, one subject can be detected in multiple directions. If there is only one target, we can simply select the direction with a stronger signal. However, when there are multiple targets, two targets may have the same rate (frequency) and therefore, we can not adopt a simple threshold to detect targets.

To address this problem, we propose a dynamic threshold-based frequency and direction scanning approach to detect targets. The key insight is, even multiple targets may have the same respiration rate, it is very unlikely for their respiration to be synchronized in time. Therefore, we can use the timing of the peaks in the curvature curves (presented in Section 4.2) to distinguish targets even with same rate and located at the same direction. We dynamically adjust the energy threshold and sequentially perform frequency scanning and direction scanning to remove false positives caused by side lobes and detect targets.

Specifically, in Frequency Scanning, we consider signals with high periodicity and large variations as candidates. We select the maximum energy among all directions in the direction-frequency spectrogram for each frequency. Figure 16 shows the result of frequency scanning for the 4 subjects whose direction-frequency spectrogram was given in Figure 15. In this example, we plot the blue line indicating the signal energy for each frequency. The maximum and minimum energy levels are 3.566 and 0.502 , and the dynamic threshold is set to $1.726{ }^{3}$ Consequently, $0.23 \mathrm{~Hz}$ and $0.37 \mathrm{~Hz}$ can be selected as candidate frequencies.

In Direction Scanning, we detect the targets from the candidates obtained in the frequency scanning step. Specifically, for a candidate frequency, the directions with energy higher than a threshold are selected as candidate directions. Here, we also calculate the dynamic threshold based on the maximum and minimum energy at this frequency. As shown in Figure 17a and Figure 18a, the peaks above the chosen threshold are candidate target directions. By using just the direction information, subject1 can be separated from subject 3 and subject4. However, when two subjects (subject3 and subject4) have similar respiration rates and are also physically close to each other, direction information is not enough to separate them. To handle this challenge scenario, we further compare

\footnotetext{
${ }^{3}$ The threshold is set to the difference between the maximum and minimum energy multiplied by an empirical constant. The frequencies with an energy higher than the threshold are selected as candidate frequencies.
} 


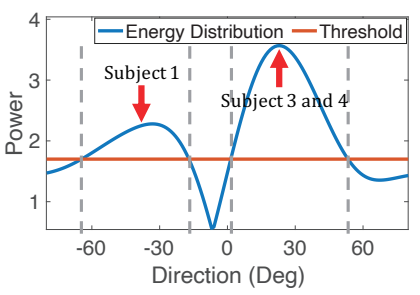

(a) Signal Power at $0.23 \mathrm{~Hz}$

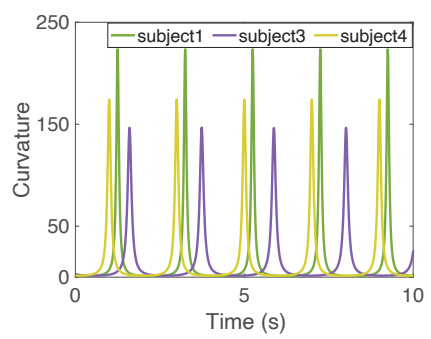

(b) Curvature clustering

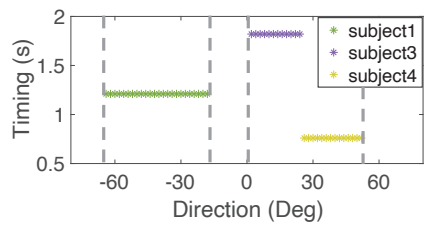

(c) Signal Timing of directions

Fig. 17. Direction scanning at $0.23 \mathrm{~Hz}$

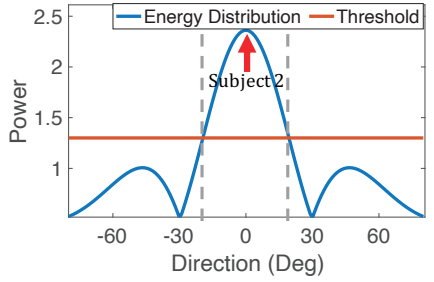

(a) Signal Power at $0.37 \mathrm{~Hz}$

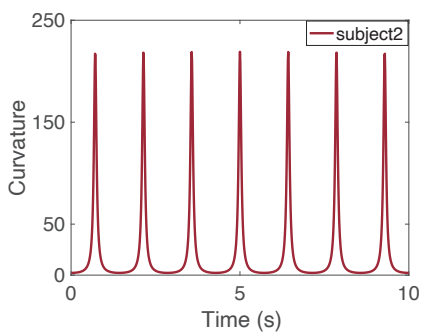

(b) Curvature

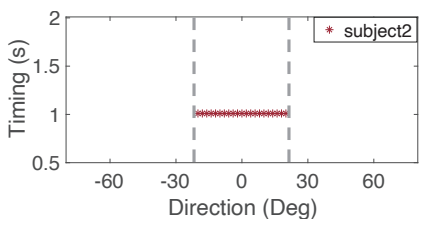

(c) Signal Timing of directions

Fig. 18. Direction scanning at $0.37 \mathrm{~Hz}$.

the timing of the peaks in the curvature lines (Section 4.2). If two candidate peaks are separated in time, it is very likely they correspond to the different targets. Therefore, even subject 3 and subject 4 are located at the same direction which can not be separated by direction information in Figure 17a, we can utilize the clear timing difference in Figure 17b to separate them. With frequency, angle and timing information, the signals from the three subjects are separated and all three subjects can be simultaneously sensed. Another subject2 is presented in Figure 18. In this case, only one target can be identified. In summary, the proposed method is able to separate subjects using angle information (different directions), frequency information (different respiration rates) and time information (different initial phases).

\section{PERFORMANCE EVALUATION}

In this section, we present experiment settings and detailed performance evaluation of the proposed multi-target sensing system.

\subsection{System Implementation and Setup}

Our LoRa-based sensing system consists of one LoRa node and one gateway. The LoRa node is a Semtech SX1276 LoRa radio [23] connected to an Arduino Uno [24]. One antenna is connected to the LoRa node. The LoRa node transmits signals at the $915 \mathrm{MHz}$ frequency band with a channel bandwidth of $125 \mathrm{kHz}$. The LoRa gateway is 
implemented on USRP X310 [25] and equipped with four commonly-used directional antennas (TX900-PB-1313). The LoRa gateway is connected to a laptop with an Intel Core i7 CPU and 16GB RAM. It collects data at a rate of 1000 samples per second per antenna and processes them using MATLAB in real time.

To monitor the respiration of multiple targets, we obtain four signal streams (one from each antenna) simultaneously. The signal streams are processed over a sliding window of $30 \mathrm{~s}$ at a time. For each steering direction, we compute the signal representations in the complex plane and apply a bandpass filter to remove outliers and high frequency noise. Next, the curvature lines are calculated to characterize human respiration. By analyzing the respiration patterns (both in frequency and time domains), we are able to determine the number of subjects and their corresponding respiration rates. A respiration monitor belt logger sensor NUL-236 [26] is employed to provide ground truth data. The ground truth respiration rate is calculated as $\frac{\text { number of breaths }}{\text { recording time }}$ in Beats Per Minute (BPM). We also develop a GUI to visualize multi-target respiration in real time, as shown in Figure 19.

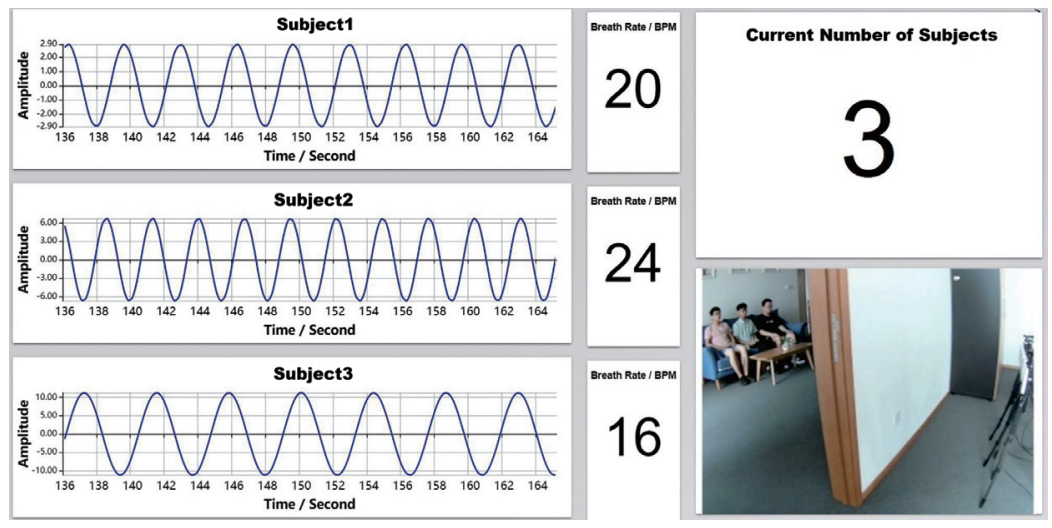

Fig. 19. Web-based user interface for LoRa sensing.

\subsection{Overall Performance}

5.2.1 Multi-target Sensing. Five subjects (3 males and 2 females) in the range of 20-60 years old were recruited to evaluate the performance of the proposed system. In this set of experiments, multiple subjects sit side-by-side in front of the LoRa transceivers at a distance of 10 meters with no obstructions and breathe naturally. The

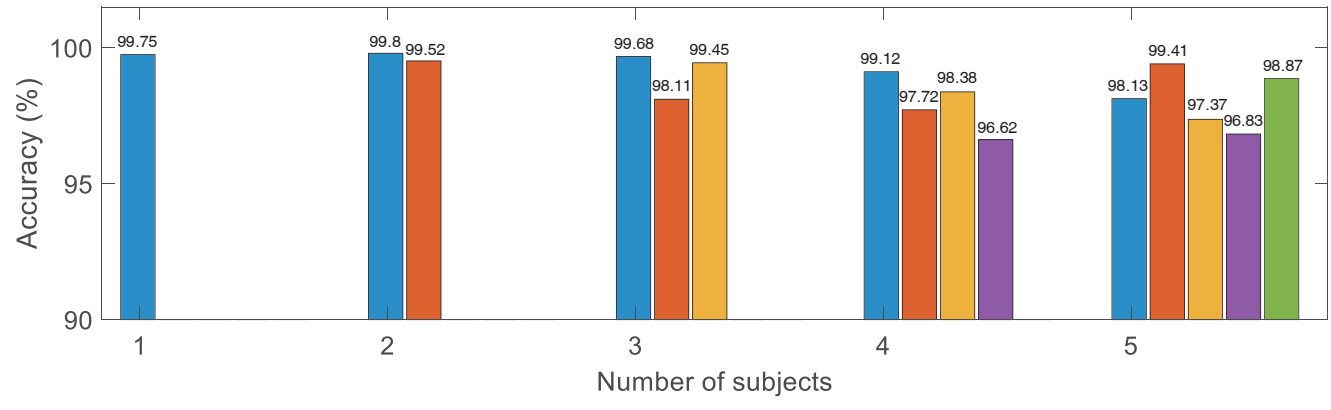

Fig. 20. Accuracy with different number of subjects. 


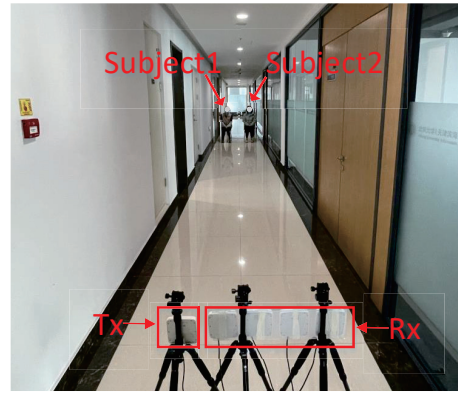

(a) corridor

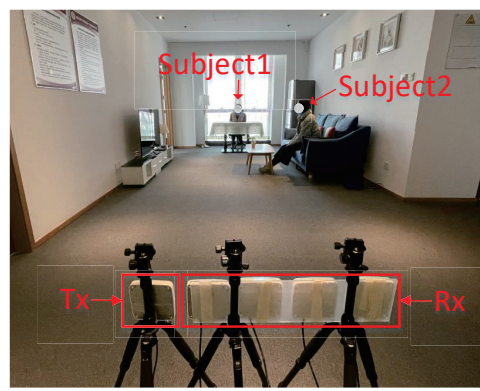

(b) Home

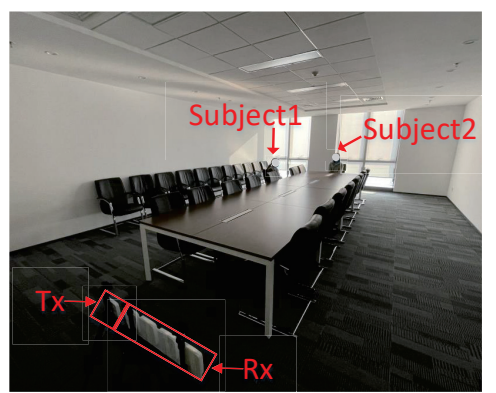

(c) Meeting room

Fig. 21. Three different experimental environments for respiration sensing.

number of subjects varies from 1 to 5 , and each experiment is repeated 5 times. In all experiments, the number of subjects is identified correctly $100 \%$ of the time. We further present the accuracy of respiration rate sensing. The accuracy is calculated as: $1-\left|\frac{\text { Estimated respiration rate-ground truth }}{\text { ground truth }}\right|$. As shown in Figure 20, when there is only a single subject, the average accuracy (among five participants) is $99.75 \%$. As the number of subjects increases, the average accuracy drops slightly. However, even for 5 subjects, the achieved average accuracy is still as high as $98.12 \%$. The accuracy for any single subject is always higher than $96 \%$.

5.2.2 Long Range Sensing. To evaluate the long-range sensing capability, we vary the distance between subjects and the transceivers from $8 \mathrm{~m}$ to $24 \mathrm{~m}$ at a step size of $4 \mathrm{~m}$. In this set of experiments, two human subjects are involved. As shown in Figure 21, we conduct experiments in three different environments: corridor, home and meeting room. Figure 22 shows that the average accuracy of respiration rate estimation of the two subjects as the distance varies. We can see that the accuracy only drops slightly from $99.62 \%$ at $8 \mathrm{~m}$ to $96.46 \%$ at $24 \mathrm{~m}$. These results demonstrate that our system can work at a long distance.

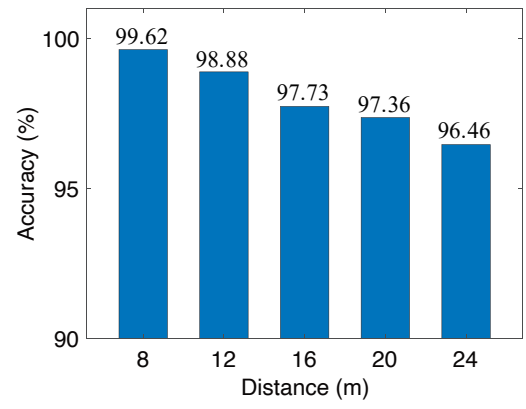

Fig. 22. Accuracy at various distances

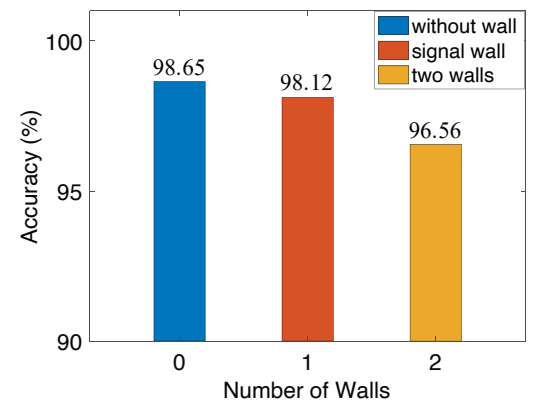

Fig. 23. Accuracy at various numbers of walls

5.2.3 Through-wall Sensing. Next, we study the sensing performance when there exist walls between the sensing device and the subjects. In this set of experiments, 3 subjects are involved and the distance between the device and subjects is $16 \mathrm{~m}$. Three subjects sit in a sofa and we evaluate the sensing accuracy when there is no wall, one wall and two walls, respectively. The wall is made of bricks with a thickness of $10 \mathrm{~cm}$. The results are shown 
in Figure 23. We can see that with walls in between, the accuracy slightly drops. This is expected because the signal gets further attenuated when there are walls. However, even with two walls in between, the accuracy is still higher than $96 \%$ at a distance of $16 \mathrm{~m}$. These results demonstrate the strong penetration capability of LoRa signals for sensing.

\subsection{Comparison with Other Sensing Technologies}

We implement three state-of-the-art sensing systems based on Wi-Fi [51], mmWave radar [53] and IR-UWB radar [7]. We compare them with the proposed LoRa-based sensing system in three aspects: 1) sensing range, 2) through-wall sensing capability, and 3) accuracy.

We implement the WiFi-based system proposed in [51] using Gigabyte mini-PC equipped with an Intel 5300 Wi-Fi card (Figure 24a). Signals are transmitted at the $5.24 \mathrm{GHz}$ frequency band. Packet transmission rates are 20 packets per second. For the mmWave-based system in [53], we employ the TI FMCW mmWave IWR1443 radar operating at $77-81 \mathrm{GHz}$ (Figure 24b). The radar is equipped with three transmission (TX) antennas and four reception (RX) antennas. We implement the IR-UWB radar-based sensing system proposed in [7] using XETHRU [54] radar module X4M05 (Figure 24c). The radar has a bandwidth of $1.4 \mathrm{GHz}$ centered at the carrier frequency of $7.3 \mathrm{GHz}$. The sampling frequency is $23.3 \mathrm{GHz}$. The frame rate is set as $400 \mathrm{~Hz}$ and one radar board is connected to a Raspberry Pi via Serial Peripheral Interface (SPI).

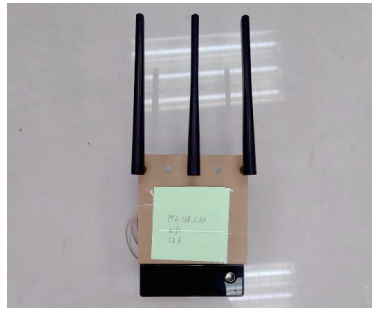

(a) WiFi device.

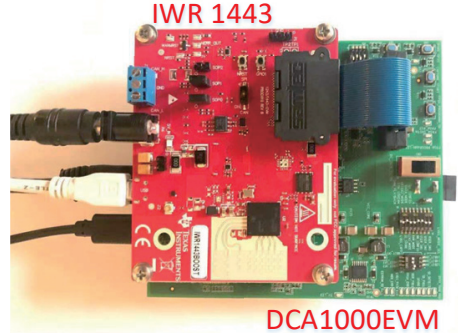

(b) FMCW mmWave Radar.

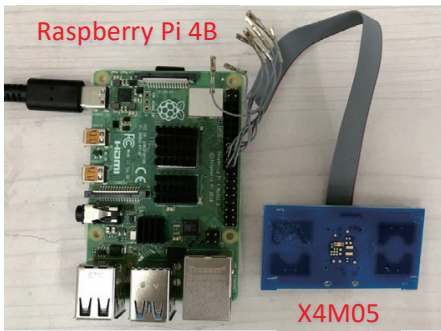

(c) IR-UWB radar.

Fig. 24. Experiment devices

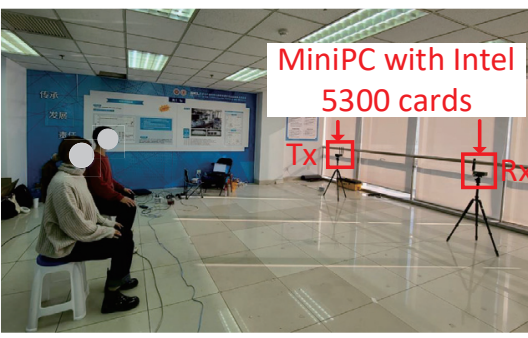

(a) WiFi deployment.

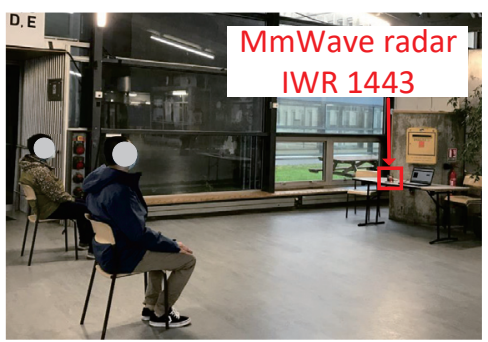

(b) FMCW Radar deployment.

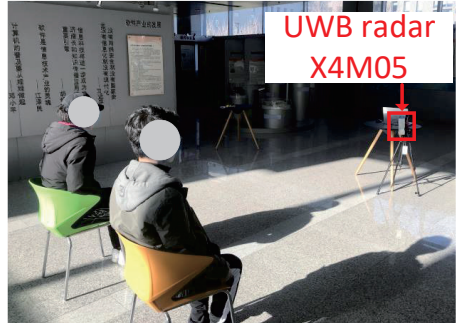

(c) IR-UWB radar deployment.

Fig. 25. All experiments are conducted in indoor environments with two targets setting in front of the different devices. 
Table 1. Comparison results of state-of-art methods

\begin{tabular}{|c|c|c|c|c|}
\hline & $\begin{array}{c}\text { Sensing } \\
\text { range }\end{array}$ & $\begin{array}{c}\text { Through } \\
\text { wall }\end{array}$ & $\begin{array}{c}\text { Target } \\
\text { number }\end{array}$ & Accuracy \\
\hline WiFi [51] & $5.5 \mathrm{~m}$ & $\times$ & $2-4$ & $0.5 \mathrm{BPM}$ \\
\hline $\begin{array}{c}\text { TI Mmwave } \\
\text { radar AWR1443 [53] }\end{array}$ & $4.3 \mathrm{~m}$ & $\times$ & 2 & $0.5 \mathrm{BPM}$ \\
\hline $\begin{array}{c}\text { XETHRU radar } \\
\text { X4M05 [7] }\end{array}$ & $5 \mathrm{~m}$ & $\times$ & $2-4$ & $0.3-0.6 \mathrm{BPM}$ \\
\hline Our system & $24 \mathrm{~m}$ & $\sqrt{ }$ & $2-5$ & $0.2-0.5 \mathrm{BPM}$ \\
\hline
\end{tabular}

The three systems are deployed in indoor environments as shown in Figure 25. We first conduct experiments to evaluate the sensing range, i.e., the maximum working distance between the target and the sensing device for each technology. Two subjects sit in front of the sensing devices and breathe naturally. The sensing ranges measured for WiFi, mmWave radar and IR-UWB are $5.5 \mathrm{~m}, 4.3 \mathrm{~m}$ and $5 \mathrm{~m}$, respectively. In contrast, our LoRa-based sensing system can achieve a much larger sensing range of $24 \mathrm{~m}$ for two targets.

To evaluate the through-wall capability, a target and a sensing device are placed in adjacent rooms separated by one wall. Surprisingly, we find that none of the systems can sense targets in a different room. This result matches the findings in [51] for WiFi-based system. For mmWave radar, the intrinsic higher carried frequency leads to a much larger attenuation, making it difficult to penetrate through walls.

Table 1 summarizes the performance of the four sensing systems. We can see that the achieved accuracy of the proposed system is comparable to the other three systems while the sensing range and penetration capability are the unique advantages.

\subsection{Robustness of the Proposed System}

In practical deployments, a number of factors may impact the performance of the proposed system, e.g., posture, body movements and similar respiration rates among subjects. We now investigate the effect of these factors on the sensing performance.

5.4.1 Impact of Different Human Postures. We consider lying and sitting, each in multiple different postures in this experiment. For lying, three postures are evaluated, i.e., on the back, on one side and face down. When sitting in a chair, subjects can have three orientations, i.e., facing the sensing device, side facing the device and back facing the device. The respiration sensing performance when the subjects are in the sitting state is shown in Figure 26. The average accuracies under the three postures are $99.62 \%, 97.56 \%$ and $96.88 \%$, respectively. The best performance is achieved when the subjects face the sensing device. This is because body displacements during respiration at the front side of the body (i.e., $4.2-5.4 \mathrm{~mm}$ ) is greater than that along the medial-lateral side (i.e., $0.6-1.1 \mathrm{~mm}$ ) and at the back side (i.e., $2.5-2.6 \mathrm{~mm}$ ) [22].

The achieved respiration sensing accuracies are high for all three postures in the lying down state as shown in Figure 27. Comparing Figure 26 and Figure 27, we note that the achieved respiration sensing accuracy is higher for lying state than that for sitting state. We believe this is because human bodies are more stable during the lying state.

5.4.2 Impact of Abnormal Respiration. We consider two types of common abnormal breathing patterns: apnea and respiration rate changes. Apnea [27] refers to a disorder during which breathing repeatedly stops and starts, typically during sleep. Respiration rate changes, e.g., tachypnea may arise from medical conditions such as pneumonia [28]. 


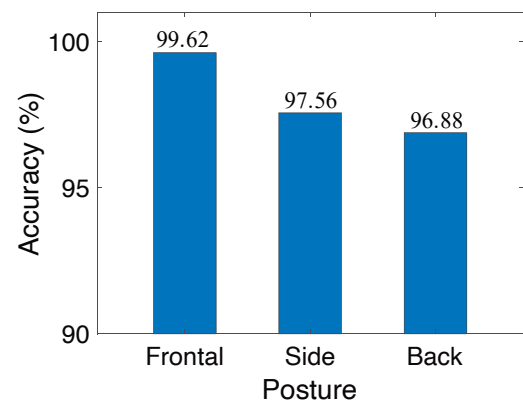

Fig. 26. Sitting scenario.

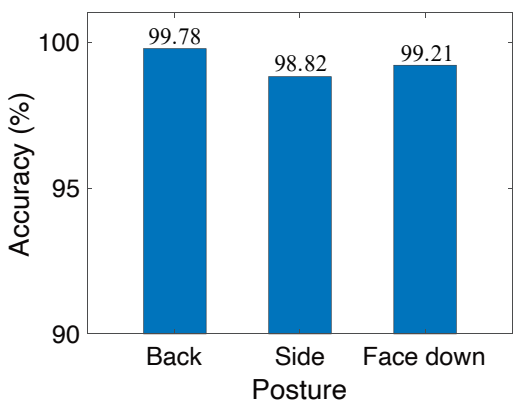

Fig. 27. Lying scenario.

In the first set of experiments, we ask one subject to mimic the apnea event by holding her/his breath for a while. Figure 28 shows the respiration pattern obtained for subject1 with breath holding (top figure) and for subject2 without breath holding (bottom figure). We can clearly see that the first subject experiences two episodes of apnea, each lasting around 20 seconds, while the breathing pattern of the second subject remains normal.

To study the effect of respiration rate variations, we mimic tachypnea by asking one subject to slow down the respiration rate for a short period, and then rapidly increase to a high rate. Figure 29 shows the extracted signal variations (blue and red lines) and the estimated respiration rates (green lines) for two subjects (one subject with tachypnea and one healthy subject). It can be seen that the respiration rate of the first subject changes from 28.17 BPM to $11.47 \mathrm{BPM}$ and then increases to $20 \mathrm{BPM}$. In contrast, the healthy subject has a steady rate (15.3 BPM). From these results, we can see the proposed system is able to detect abnormalities in respiration, which is useful in applications such as sleep apnea detection.
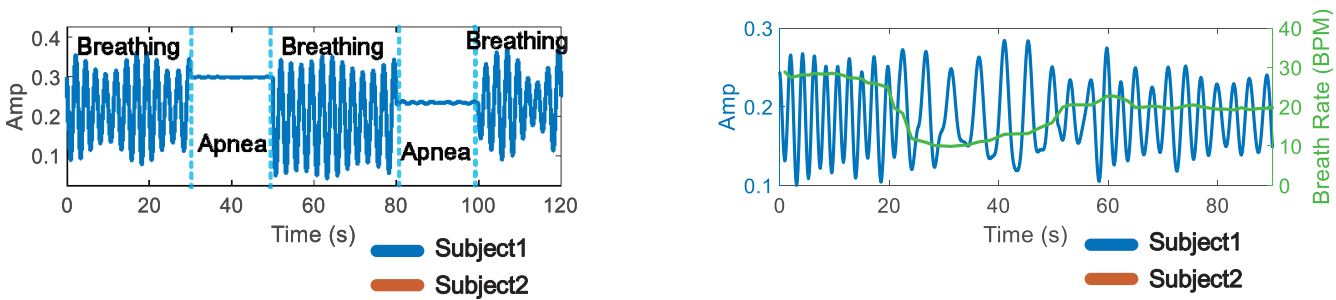

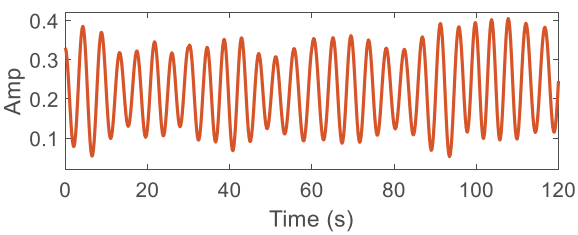

Fig. 28. Impact of partial apnea while lying.

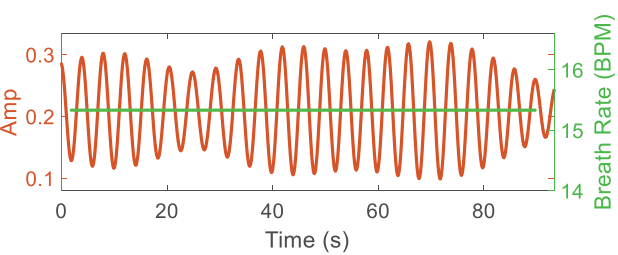

Fig. 29. Impact of respiration rate change.

5.4.3 Impact of Similar Respiration Rates. Recall in Section 4.2 we utilize curvatures to differentiate two subjects with similar respiration rates. To validate this idea, we ask two subjects to breathe at a rate of 14 BPM following a metronome. Figure 30 shows the obtained respiration waveforms for both subjects. Even two subjects have the same breathing rate, we can still use the peak point locations to differentiate the two subjects. 


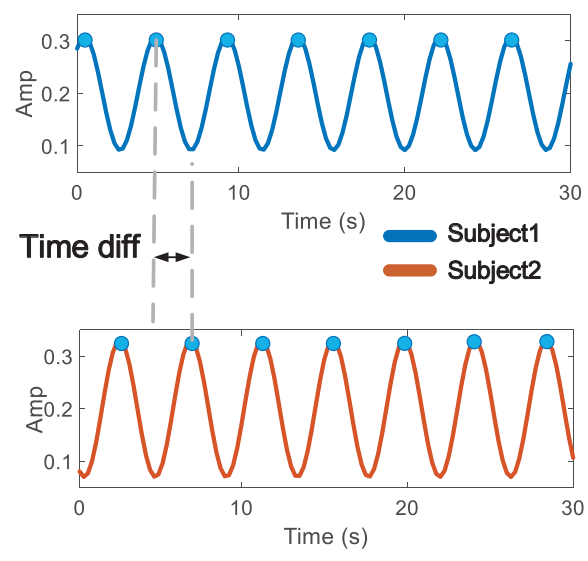

Fig. 30. Subjects with similar respiration rates.
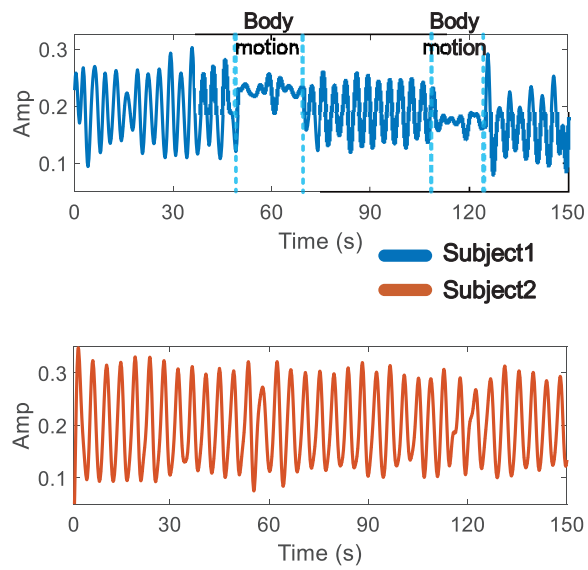

Fig. 31. Impact of body movements.

5.4.4 Impact of Body Movements. In the last set of experiments, we investigate the impact of body movements in multi-target respiration sensing. Two subjects are asked to sit next to each other in front of the sensing device. One subject breathes steadily without body movements. The other subject performs two series of activities in sequence: i) stands up from a chair and then sits down, and ii) picks up a smartphone from a table and then puts it back. Figure 31 shows the obtained respiration waveforms of the two subjects. It can be seen that when the first subject undergoes body motions, the respiration-induced pattern disappears by employing the bandpass filtering. The respiration pattern resumes as soon as the subject becomes stationary again. Interestingly, despite the close proximity of the two subjects, body movements of the first subject has negligible impact on the respiration sensing of the second subject - slight distortion in the waveform can be observed but respiration rate estimation is still accurate. This result once again demonstrates the effectiveness of LoRa beamforming in separating signals from different subjects.

\section{RELATED WORK}

\subsection{LoRa-based Communication and Sensing.}

Most existing research on LoRa focuses on long-distance communication and IoT data transmissions. Semtech's white paper [34] shows that LoRa networks could provide efficient IoT data transmissions for smart cities. LoRa technologies can find applications in many scenarios such as environment monitoring [36] [37], facility management [38], intelligent building control [39], vehicle tracking [40], and smart lighting [35]. For example, Ke et al. deploy LoRa nodes in building corridors to form a mesh network and transfer collected sensor data for campus monitoring. Mathur et al. [40] utilize LoRaWAN to enable crowdsourced traffic sensing. They propose an adaptive data aggregation and re-transmission scheme for relaying traffic data from sensors. Ali et al. [41] propose to utilize a sensing platform with LoRa radios to monitor climate changes. All these works only employ LoRa transceivers to transfer collected sensor data. Recently, LoRa-based localization has gained some interests. Gu et al. [42] study the feasibility of LoRa-based localization, which shows a phase-based localization approach outperforms RSSI-based and TDOA-based approaches. Chen et al. [43] utilize LoRa signals for contactless localization with the help of drone mobility to carry the LoRa transceivers and move around the target area to perform wireless sensing. Rajalakshmi et al. [44] develop a multi-band LoRa backscatter system for localization, reaching a range up to $60 \mathrm{~m}$. The recent work by Islam et al. [45] compares LoRa with two other popular wireless technologies (BLE 
and WiFi) for indoor localization. Considering coverage, stability, accuracy, power consumption, and cost, they conclude that LoRa is a suitable solution for indoor localization, especially in large indoor space like warehouses and multi-storied buildings.

\subsection{Multi-target Respiration Sensing Using Wireless Signals.}

Many wireless technologies have been employed for multi-target respiration sensing include commodity WiFi [49][30], RFID [43] and dedicated FMCW radars [50][53], 60GHz radars [31], and UWB radars [33]. Chen et al. [49] utilize a frequency analysis method on WiFi CSI signal to infer the respiration rates of multiple persons. Wang et al. [30] leverage the canonical polyadic (CP) decomposition technique to handle the CSI phase difference and obtain multi-target breathing signals. However, the computational complexity of such method is high, hindering its application in real-time monitoring. Special-purpose device such as FMCW radars, $60 \mathrm{GHz}$ radars and UWB radars have also been explored in multi-target respiration monitoring. Ahmad et al. [53] utilize range-azimuth resolution point cloud to distinguish multiple targets using TI FMCW radars. However, the working range of this radar is still small (only $4.3 \mathrm{~m}$ ), and the hardware cost. Santra et al. [31] present a compact short-range $60 \mathrm{GHz}$ radar system by adopting Infineon's $60 \mathrm{GHz}$ radar chipset. The chirp can sweep a large bandwidth (up to $7 \mathrm{GHz}$ ). The maximum detectable range is still limited to $4 \mathrm{~m}$. Duo to the large signal attenuation, these works are limited to to work only in direct line-of-sight scenarios. The proposed system in contrast can work at a long distance as well as through walls. Our previous work [46] develops a LoRa-based system that is capable of sensing both coarse-grained and fine-grained human activities at a distance through walls. However, the approach is limited to single target scenario. In [48], the authors propose the virtual fence concept to enable multi-target sensing. Though promising, the virtual fence method requires to know the target's direction information which may not be available in real-world environment. FarSense [47] is a WiFi-based respiration sensing system which employs two antennas to eliminate the common noise to achieve a larger sensing range. Although the respiration sensing range is increased to 8 meters, it is still much smaller than our proposed LoRa system. The sensing range is $3 \times$ larger than that achieved in FarSense.

\section{DISCUSSION}

In this section, we briefly discuss the limitations and potential future work.

\subsection{Further Increasing the Number of Targets Being Sensed.}

With a four-antenna array in our current implementation, we successfully demonstrated that the proposed system can simultaneously sense five targets spatially located as shown in Fig. 20. We believe 5-target is not the limit of the proposed system with four antennas. As the proposed system utilizes not just the space domain (angle) information but also the frequency domain (respiration rate) and time domain (phase) information to distinguish targets, even the targets are located at similar directions, there are still chances we can distinguish them in the frequency domain and time domain. From our experiments, the most important parameter affecting the number of targets capable of being sensed is the size of the antenna array. With more antennas, the spatial angle resolution can be further increased to simultaneously sense even more targets.

\subsection{Practical Usage Scenarios.}

We believe the proposed LoRa sensing techniques can be applied to a large variety of real-life scenarios. Besides the traditional smart home scenario which is feasible with WiFi sensing, we envision the proposed system can enable new application scenarios which are not possible with short-range WiFi or radar sensing. We briefly describe two example usage scenarios below: 1) In hospital scenario, the patients with infectious virus such as COVID-19 can be contactlessly monitored through walls. This will help provide increased protection for health 
care workers; 2) In disaster relief efforts after earthquakes, survivors may be deeply buried under debris. The strong penetration capability of LoRa sensing can help to detect survivors in this challenging scenario.

\section{CONCLUSION}

In this paper, we develop novel techniques to enable long-range multi-target LoRa sensing. To sense multiple targets simultaneously, we propose a receiver-side LoRa beamforming approach. We efficiently utilize not just the angle information but also the frequency and timing information to help differentiate targets. We demonstrate the capability of simultaneously sensing five targets at a high accuracy and believe this number can be further increased. We believe the long range and strong penetration capabilities of LoRa sensing moves wireless sensing one step closer to real-life adoption. We envision more exciting applications can be enabled on top of the proposed LoRa sensing platform.

\section{ACKNOWLEDGMENTS}

This work is partially supported by the National Natural Science Foundation of China (No. 61802373, No. 62072450), the Youth Innovation Promotion Association, Chinese Academy of Sciences (No. 2020109), the National Key Research and Development project of China under Grant (No. 2020YFB2103900), the Labex DigiCosme (project ANR11LABEX0045DIGICOSME) operated by ANR as part of the program «Investissement d'Avenir $\gg$ Idex ParisSaclay (ANR11IDEX000302), the EU CHIST-ERA RadioSense Project, the EU Horizon 2020 research and innovation programme IDEA-FAST (No. 853981), the Discovery Grant from Natural Sciences and Engineering Research Council of Canada and the Google European Doctoral Fellowship in Wireless Networking.

\section{REFERENCES}

[1] D. Zhang, H. Wang, and D. Wu, "Toward Centimeter-Scale Human Activity Sensing with Wi-Fi Signals," IEEE Computer, vol. 50, pp. 48-57, 2017.

[2] F. Zhang, D. Zhang, J. Xiong, H. Wang, K. Niu, B. Jin, and Y. Wang. 2018. From Fresnel Diffraction Model to Fine-grained Human Respiration Sensing with Commodity Wi-Fi Devices. Proc. ACM Interact. Mob. Wearable Ubiquitous Technol., vol. 2, no. 1, Article 53, 2018.

[3] L. Chen, J. Xiong, X. Chen, S. Lee, D. Zhang, T. Yan, and D. Fang. 2019. LungTrack: Towards Contactless and Zero Dead-Zone Respiration Monitoring with Commodity RFIDs. Proc. ACM Interact. Mob. Wearable Ubiquitous Technol., vol. 3, no. 3, Article 79, 2019.

[4] J. Wang, J. Xiong, X. Chen, H. Jiang, R. K. Balan, and D. Fang, "TagScan: Simultaneous Target Imaging and Material Identification with Commodity RFID Devices," in Proceedings of The 23rd Annual International Conference on Mobile Computing and Networking (Mobicom 2017), pp. 288-300, 2017.

[5] T. Kao, J. Lin. 2013. Vital sign detection using 60-GHz Doppler radar system. In 2013 IEEE International Wireless Symposium (IWS). IEEE, 1-4.

[6] $60 \mathrm{GHz}$ radar, 2016, https://www.infineon.com/cms/en/product/promopages/60GHz/.

[7] Y. Yang, J. Cao, X. Liu and X. Liu, "Multi-Breath: Separate Respiration Monitoring for Multiple Persons with UWB Radar," 2019 IEEE 43rd Annual Computer Software and Applications Conference (COMPSAC), 2019, pp. 840-849.

[8] B. Anderson, M. Shi, V. Y. F. Tan, and Y. Wang, "Mobile Gait Analysis Using Foot-Mounted UWB Sensors," Proc. ACM Interact. Mob. Wearable Ubiquitous Technol, vol. 3, no. 3, Article 73, 2019.

[9] F. Zhang, Z. Wang, B. Jin, J. Xiong, and D. Zhang, "Your Smart Speaker Can "Hear" Your Heartbeat!" Proc. ACM Interact. Mob. Wearable Ubiquitous Technol. vol. 4, no.4, 24 pages, 2020.

[10] T. Wang, D. Zhang, Y. Zheng, T. Gu, X. Zhou, and B. Dorizzi. 2017. C-FMCW Based Contactless Respiration Detection Using Acoustic Signal. PACM Interact. Mob. Wearable Ubiquitous Technol. 1, 4, Article 170, 2017.

[11] T. Li, C. An, Z. Tian, A. Campbell, and X. Zhou, "Human Sensing Using Visible Light Communication," In Proceedings of the 21st Annual International Conference on Mobile Computing and Networking (MobiCom 2015), pp. 331-344, 2015.

[12] Y. Li, T. Li, R. Patel, X. Yang, and X. Zhou. 2018. Self-Powered Gesture Recognition with Ambient Light. In Proceedings of the 31st Annual ACM Symposium on User Interface Software and Technology (UIST 2018). pp. 595-608, 2018.

[13] Dakulagi, V., Alagirisamy, M. Adaptive Beamformers for High-Speed Mobile Communication. Wireless Wireless Personal Communications volume, 1691-1707 (2020). 
[14] A. Augustin, J. Yi, T. Clausen, and W. Townsley. 2016. A study of LoRa: Long range and low power networks for the internet of things. Sensors 16, 9 (2016), 1466.

[15] A. Hoeller, R. D. Souza, O. L. Alcaraz López, H. Alves, M. de Noronha Neto and G. Brante, "Analysis and Performance Optimization of LoRa Networks With Time and Antenna Diversity," in IEEE Access, vol. 6, pp. 32820-32829, 2018.

[16] K. Niu, F. Zhang, J. Xiong, X. Li, E. Yi, and D. Zhang, "Boosting fine-grained activity sensing by embracing wireless multipath effects," in Proceedings of the International Conference on emerging Networking EXperiments and Technologies (CoNEXT 2018), pp. 139-151, 2018.

[17] H. Li, A. Shrestha, H. Heidari, J. Le Kernec and F. Fioranelli, "Bi-LSTM Network for Multimodal Continuous Human Activity Recognition and Fall Detection," IEEE Sensors Journal, vol. 20, pp. 1191-1201, 2020.

[18] Z. Meng, S. Fu, J. Yan, H. Liang, A. Zhou, S. Zhu, H. Ma, J. Liu, N. Yang, "Gait Recognition for Co-Existing Multiple People Using Millimeter Wave Sensing," in Proceedings of the AAAI Conference on Artificial Intelligence, vol. 34, no. 1, 2020.

[19] F. Zhang, K. Niu, J. Xiong, B. Jin, T. Gu, Y. Jiang, and D. Zhang, "Towards a diffraction-based sensing approach on human activity recognition," in Proceedings of the ACM on Interactive, Mobile, Wearable and Ubiquitous Technologies, vol. 3, no. 1, pp. 1-25, 2018.

[20] https://www.semtech.com/uploads/documents/WiFi_Immunity_App_Notes.pdf

[21] https://www.semtech.com/uploads/documents/DS_SX1276-7-8-9_W_APP_V6.pdf(2019)

[22] C. Lowanichkiattikul, M. Dhanachai, C. Sitathanee, S. Khachonkham, and P. Khaothong, "Impact of chest wall motion caused by respiration in adjuvant radiotherapy for postoperative breast cancer patients," SpringerPlus 5, Article 144, 2016.

[23] Semtech SX1276 Transceiver. https://www.semtech.com/products/wireless-rf/lora-transceivers/sx1276.

[24] Arduino. https://store.arduino.cc/usa/arduino-uno-rev3.

[25] USRP X310. https://www.ettus.com/all-products/x310-kit/.

[26] Respiration Monitor Belt logger sensor NUL-236. https://neulog.com/respiration-monitor-belt/.

[27] WebMD. https://www.webmd.com/sleep-disorders/sleep-apnea/understanding-obstructive-sleep-apnea-syndrome.

[28] L. Gattinoni, D. Chiumello, P. Caironi, M. Busana, F. Romitti, L. Brazzi and L. Camporota, COVID-19 pneumonia: different respiratory treatments for different phenotypes?. Intensive Care Med 46, 1099-1102 (2020).

[29] B. K. Jennison. 2000. Performance of a linear frequency-modulated signal detection algorithm. In Record of the IEEE 2000 International Radar Conference. IEEE, 447-450.

[30] X. Wang, C. Yang, and S. Mao, "TensorBeat: Tensor Decomposition for Monitoring Multiperson Breathing Beats with Commodity WiFi," ACM Trans. Intell. Syst. Technol. 9, 1, Article 8 (October 2017), 27 pages.

[31] A. Santra, R. V. Ulaganathan, T. Finke, A. Baheti, "Short-range multi-mode continuous-wave radar for vital sign measurement and imaging," in Proceedings of 2018 IEEE Radar Conference (RadarConf18), pp. 946-950, 2018.

[32] Mahfoudi, MN, Sivadoss, G, Korachi, OB, Turletti, T, Dabbous, W. Joint range extension and localization for low-power wide-area network. Internet Technology Letters. 2019

[33] Y. Yang, J. Cao, X. Liu, and X. Liu, "Multi-Breath: Separate Respiration Monitoring for Multiple Persons with UWB Radar," in Proceedings of the 2019 IEEE 43rd Annual Computer Software and Applications Conference (COMPSAC), vol. 1, pp. 840-849, 2019.

[34] Semtech, "Smart cities transformed using semtech's LoRa technology," white paper, 2017.

[35] Semtech, "Smart lighting," https://www.semtech.com/lora/lora-applications, WhitePaper.

[36] S. Liu, C. Xia, and Z. Zhao, "A low-power real-time air quality monitoring system using lpwan based on lora," in Proceedings of the 13th IEEE International Conference on Solid-State and Integrated Circuit Technology (ICSICT), pp. 379-381, 2016.

[37] T. Ameloot, P. V. Torre, and H. Rogier, "LoRa indoor performance: an office environment case study," in Proceedings of the International Applied Computational Electromagnetics Society Symposium, pp. 1-2, 2018.

[38] P. Sommer, Y. Maret, and D. Dzung, "Low-Power Wide-Area Networks for Industrial Sensing Applications," in Proceedings of the 2018 IEEE International Conference on Industrial Internet (ICII), pp. 23-32, 2018.

[39] K. H. Ke, Q. W. Liang, G. Zeng, J. Lin, and H. Lee, "A LoRa wireless mesh networking module for campus-scale monitoring: demo abstract," in Proceedings of the 16th ACM/IEEE International Conference on Information Processing in Sensor Networks (IPSN), pp. 259-260, 2017.

[40] S. Mathur, A. Sankar, P. Prasan, and B. Iannucci, "Energy Analysis of LoRaWAN Technology for Traffic Sensing Applications," in Proceedings of the Intelligent Transportation Society of America (ITS) World Congress, vol. 29, 2017.

[41] N. A. Azmi Ali and N. A. Abdul Latiff, "Environmental Monitoring System Based on LoRa Technology in Island," in Proceedings of the 2019 IEEE International Conference on Signals and Systems (ICSigSys), pp. 160-166, 2019.

[42] C. Gu, L. Jiang, and R. Tan, "LoRa-Based Localization: Opportunities and Challenges," in Proceedings of the 2019 International Conference on Embedded Wireless Systems and Networks, pp. 413-418, 2019.

[43] L. Chen, J. Xiong, X. Chen, S. I. Lee, K. Chen, D. Han, D. Fang, Z. Tang, and Z. Wang, "WideSee: towards wide-area contactless wireless sensing," in Proceedings of the 17th Conference on Embedded Networked Sensor Systems (SenSys 2019), pp. 258-270, 2019.

[44] Rajalakshmi Nandakumar, Vikramlyer, ShyamnathGollakota, "3D Localization for Sub-Centimeter Sized Devices," in Proceedings of the 16th ACM Conference on Embedded Networked Sensor Systems, pp. 108-119, 2018.

[45] B. Islam, M. T. Islam, and S. Nirjon, "Feasibility of LoRa for Indoor Localization," on-line, from semanticscholar.org, pp. 1-11, 2017.

Proc. ACM Interact. Mob. Wearable Ubiquitous Technol., Vol. 5, No. 2, Article 85. Publication date: June 2021. 
[46] F. Zhang, Z. Chang, K. Niu, J. Xiong, B. Jin, Q. Lv, and D. Zhang. 2020. Exploring LoRa for Long-range Through-wall Sensing. Proc. ACM Interact. Mob. Wearable Ubiquitous Technol. 4, 2, 2020

[47] Y. Zeng, D. Wu, J. Xiong, E. Yi, R. Gao, D. Zhang. 2019. FarSense: Pushing the Range Limit of WiFi-based Respiration Sensing with CSI Ratio of Two Antennas. Proc. ACM Interact. Mob. Wearable Ubiquitous Technol. 3, 3, Article 121, 2019.

[48] B. Xie, J. Xiong. 2020. Combating interference for long range LoRa sensing. Proceedings of the 18th Conference on Embedded Networked Sensor Systems, 69-81, 2020.

[49] C. Chen, Y. Han, Y. Chen, H. Lai, F. Zhang, B. Wang, and K.J.R. Liu, "TR-BREATH: Time-Reversal Breathing Rate Estimation and Detection," IEEE Transactions on Bio-medical Engineering, vol. 65, no. 3, pp. 489-501, 2018.

[50] F. Adib, H. Mao, Z. Kabelac, D. Katabi, and R. C. Miller, "Smart Homes that Monitor Breathing and Heart Rate," in Proceedings of the 33rd Annual ACM Conference on Human Factors in Computing Systems (CHI 2015), pp. 837-846, 2015.

[51] Youwei Zeng, Dan Wu, Jie Xiong, Jinyi Liu, Zhaopeng Liu, and Daqing Zhang. 2020. MultiSense: Enabling Multi-person Respiration Sensing with Commodity WiFi. Proc. ACM Interact. Mob. Wearable Ubiquitous Technol. 4, 3, Article 102 (September 2020 ), 29 pages.

[52] Nan Yu, Wei Wang, Alex X Liu, and Lingtao Kong. 2018. QGesture: Quantifying Gesture Distance and Direction with WiFi Signals. Proceedings of the ACM on Interactive, Mobile, Wearable and Ubiquitous Technologies 2, 1 (2018), 51.

[53] A. Ahmad, J. C. Roh, D. Wang and A. Dubey, "Vital signs monitoring of multiple people using a FMCW millimeter-wave sensor," 2018 IEEE Radar Conference (RadarConf18), 2018, pp. 1450-1455.

[54] Xethru impulse uwb radar chip, https://www.xethru.com/x4-radarchip.html/, accessed: 2018-03-30.

[55] J. C. Spall. Introduction to stochastic search and optimization: estimation, simulation, and control. John Wiley and Sons, 2005. 